44th AIAA Aerospace Sciences Meeting and Exhibit

9-12 January, 2006/Reno, NV

\title{
Statistical Characterization of Environmental Error Sources Affecting Electronically Scanned Pressure Transducers
}

\author{
Del L. Green, ${ }^{*}$ Eric L. Walker, ${ }^{\dagger}$ and Joel L. Everhart ${ }^{\ddagger}$ \\ NASA Langley Research Center, \\ Hampton, VA 23681
}

\begin{abstract}
Minimization of uncertainty is essential to extend the usable range of the 15-psid Electronically Scanned Pressure (ESP) transducer measurements to the low free-stream static pressures found in hypersonic wind tunnels. Statistical characterization of environmental error sources inducing much of this uncertainty requires a well defined and controlled calibration method. Employing such a controlled calibration system, several studies were conducted that provide quantitative information detailing the required controls needed to minimize environmental and human induced error sources. Results of temperature, environmental pressure, over-pressurization, and set point randomization studies for the 15-psid transducers are presented along with a comparison of two regression methods using data acquired with both 0.36-psid and 15-psid transducers. Together these results provide insight into procedural and environmental controls required for long term high-accuracy pressure measurements near 0.01 psia in the hypersonic testing environment using 15-psid ESP transducers.
\end{abstract}

\section{Introduction}

Electronically Scanned Pressure (ESP) transducers (Figure 1) are used when many simultaneous pressure measurements are required at different locations. The System $8400^{1}$ manufactured by Pressure Systems Inc., utilizes these multiplexed multi-port transducer modules as part of a differential pressure acquisition system. This system also includes pressure calibration hardware, along with data acquisition and data reduction software. ESP transducers can be accurate to manufacturer specifications when used in controlled environments (i.e., when environmental sources of error are controlled) or when changes in the measurement system environment can be minimized by rapid in-situation (in-situ) calibrations, an on-demand capability designed into the System $8400^{1}$ measurement system. However, significant errors may be introduced into pressure measurements by transducer temperature instabilities, changes in module environmental pressure, vibratory loading, and transducer over-pressurization if in-situ calibrations are precluded.

Validation of advanced computational fluid dynamics (mathematical) models and the requirement to obtain precise aerodynamic loads from wind tunnel tests have driven improvements in transducer technology toward more accurate measurements with quantifiable uncertainties. The hypersonic aerothermodynamic testing environment offers particularly stringent conditions such as low free-stream static pressure and high temperatures that often vary over short time periods. These short term variations coupled with operational constraints imposed by these types of research facilities provide error sources and increase pressure measurement uncertainty, sometimes outside the manufacturer's specifications. Short wind-on run durations of

*Aerospace Systems Analyst. Aeronautics Systems Analysis Branch. Systems Analysis \& Concepts Directorate. NASA Langley Research Center. Member AIAA.

${ }^{\dagger}$ Research Engineer. Configuration Aerodynamics Branch. Aerodynamics, Aerothermodynamics, and Acoustics Competency. NASA Langley Research Center. Student Member AIAA.

${ }^{\ddagger}$ Senior Research Engineer. Aerothermodynamics Branch. Aerodynamics, Aerothermodynamics, and Acoustics Competency. NASA Langley Research Center. Senior Member AIAA.

This material is declared a work of the U.S. Government and is not subject to copyright protection in the United States.2006 
blow-down tunnels, usually less than 5 minutes, eliminate the ability to conduct true in-situ calibrations due to the long settling times required for low-pressure set points.

The low free-stream static pressures, typically near 0.02 psia in the NASA Langley 31-Inch Mach 10 Tunnel, require that the differential ESP transducers be used as absolute gages by evacuating the transducer reference to vacuum. Air crossing between the higher-pressure external environment and the internal vacuum reference through seal leaks result in a pressure drop in the reference pressure when the wind tunnel is evacuated, immediately prior to the test run, from atmospheric pressure to a vacuum. These operational conditions coupled with tunnel operation procedures induce measurement uncertainty due to unstable reference pressures. Temperature changes are also recognized as a major time-dependent source of error for ESPs, causing transducers to shift away from the calibration response obtained at a different level of temperature. Due to the these adverse conditions, environmental control of the ESP modules is required to ensure temperature and reference pressure stability minimizing measurement error normally mitigated by in-situ calibrations.

Another issue related to the use of ESPs in the hypersonic environment is the wide range of pressures measured on some types of models. Pressure measurements on powered models are especially problematic due to the possibility that surface pressures at a prescribed location may range over two orders of magnitude. One may ask, is a lower range transducer appropriate for lower pressures under these circumstances? Porting multiple transducers to a single pressure orifice is problematic due to increased lag time required equalizing measured pressures as well as doubling the required transducer count. Over-pressurization of the 0.36psid transducer is also common under these conditions. The over-pressurization changes the zero pressure voltage response invalidating the transducer calibration. To mitigate the powered model problem, a large range pressure transducer with an accurate low-pressure measurement capability is required.

For these conditions, minimizing human influences on measurement accuracy is critical, requiring well developed procedures to be implemented for consistency. Characterization of environmental and procedural error sources is also required to identify the proper controls needed to ensure that accuracy is not compromised. This paper addresses a few of the environmental, procedural, and instrument sources which contribute to low-pressure measurement error. These sources of error are characterized using Statistical Process Control $^{2}$ (SPC) analysis methods. The error characterization allows the determination of whether the error sources need to be controlled, mitigated, or neglected.

The calibration discussed was used to conduct four studies. These studies were analyzed using SPC to characterize the influence of transducer temperature, module environmental pressure, calibration set-point randomization, and transducer over-pressurization on measurement uncertainty. The significance of the environmental and procedural effects will be used to determine the controls needed to maintain reproducible calibrations over time. Along with the environmental and procedural error analysis, two regression models have been analyzed to assess curve fit error, calibration stability, and determine the best regression model. This paper addresses the following fundamental questions:

- What is the appropriate regression model to minimize transducer calibration error when used under absolute conditions?

- How sensitive are the ESP transducers to temperature fluctuations?

Are the calibration coefficients sensitive to temperature change?

- Does the transducer calibration change when the environmental pressure changes?

- Is randomization of set points required to improve transducer calibration accuracy?

- Does over-pressurization of an ESP transducer require a new calibration?

- What should the re-calibration time interval be?

- Can the 15-psid transducer be calibrated to measure pressures down to 0.01 psia?

This paper demonstrates how adequate environmental and procedural control will provide sufficient error reduction and enable 15-psid ESP transducers to measure pressures near 0.01 psia, facilitating their use and minimizing the potential of transducer over-pressurization during hypersonic wind tunnel testing. The procedural and environmental controls are provided by a calibration system described in reference ${ }^{3}$ and discussed briefly in Section II. 


\section{Experimental and Analysis Method}

Before presenting the experimental results, the Statistical Process Control analysis method must be discussed along with a description of the calibration system used to conduct the experiment. Figures illustrating the reproducibility of the calibration system will be used as examples when describing the SPC analysis method.

\section{A. Statistical Process Control Analysis Method}

Figure 2 is a three-way chart used to analyze the averaged voltage responses and will be used as an illustrative example of the SPC analysis method. First, however, some terms must be defined. A calibration "run" consists of acquired pressure and voltage measurement data at each calibration set pressure or calibration point. The term "index" will be used when data are plotted from more than one study, where run numbers are discontinuous.

Each point on the averages or $\bar{X}$ chart (top) is calculated using the average of 24 settled $^{\text {a }}$ ESP voltage measurements $(X)$ obtained at the prescribed set pressure (point) over 48 seconds. The $\bar{X}$ (or mean) values calculated from data obtained at each calibration set point will be designated as the $V$ values used in the regression discussion later. Each averaged voltage value is plotted in chronological order of acquisition which is normally the run number. The average chart is used to determine whether the system is operating in a statistically controlled manner by comparing relative magnitude of mean voltage values from each calibration relative to control limits based on the mean value of the moving range discussed below.

Two levels of variation are illustrated in a three-way chart. First, the Moving Range Chart depicted in figure 2 illustrates the variation found between the data groups and shows the relative variation between calibration runs. The second level is illustrated by the Range Chart. The Range Chart (bottom) is plotted using the range $(R)$ of the values found within each data group, which in this case are the voltage values, used to calculate the $\bar{X}$ value. The equation used to calculate the "range" is shown below as Equation 1.

$$
R=X_{\max }-X_{\min } .
$$

Range values illustrated in figure 2 describe the magnitude of voltage variation contained within each $\bar{X}$ data point. The moving range chart (Middle) is generated using the calculated moving range $(m R)$ or absolute value of the differential between the adjacent $\bar{X}$ values. This chart is used to assess between group or run-torun variation and used to determine the control limits. Once the charts are plotted, the centerline (CL) and the upper and lower control limits (UCL) and (LCL) denoted together below as ( $\left.{ }^{*} \mathrm{CL}\right)$ are calculated for each of the sub charts using the chart specific equations presented by Wheeler. ${ }^{5}$ Note that figure 2 has control limits plotted based on both the range and the moving range values for descriptive purposes. Normally only the moving range limits plotted on a three-way chart.

Figure 2 shows a process that appears to be in statistical control, relative to the moving range based control limits $\left({ }^{*} \mathrm{CL}_{\bar{X}}(m R)\right)$, because the points all reside inside the control limits. Notice that the system appears to be out of statistical control relative to the range based control limits $\left({ }^{*} \mathrm{CL}_{\bar{X}}(R)\right)$. SPC can not distinguish whether the control limits are too narrow or broad; that assessment must be made by the process user.

Another chart is used to analyze individual values. The Individuals and Moving Range (XmR) Chart is created very similar to the three way chart discussed previously. The individuals chart is plotted using the individual values and the moving range is plotted as before. The Range chart is not created because there is no range within the individual value. All control limit calculations are conducted using the equations presented by Wheeler. ${ }^{5}$ Examples of this chart will be seen repeatedly in Section III. Most of the charts illustrated within this paper are XmR Charts analyzing calibration coefficients. Zero Pressure Response voltages denoted later as $V_{0}$ are plotted on XmR Charts for purposes of consistency.

A predictable system will vary randomly about the centerline of the data in the averages chart and within the control limits. Points that fall outside the control limits are signals of an unstable system and should be investigated. The control limits are generated using the mean range or mean moving range values, which allow the estimation of the standard deviation. This mean range value is adjusted by a coefficient that scales the limits based on the number of values used to determine the mean range.

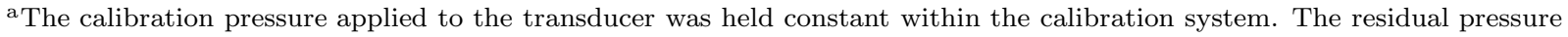
was allowed time to stabilize which in turn allowed the measured response voltage to stabilize. Details of this process can be found in Green $^{4}$
} 


\section{B. Calibration System}

The calibration system, ${ }^{3}$ described and characterized in detail in Green ${ }^{4}$ was used to conduct these experiments. The pressure controls consist of Proportional Integrating and Derivative (PID) pressure control capable of maintaining pressure to within $1 \%$ of the set pressure. Calibration pressures are measured by a separate system of transducers from the pressure control. The measurement transducers are accurate to within 0.00003 psid from 0.001 to 1 psid and 0.00075 psid from 1 to 15 psid. Temperature is controlled within 0.1 degree $\mathrm{F}$ from the set point. The transducers environmental pressure is maintained within an Environmental Control Chamber (ECC) which was described in reference 3 and in detail in reference 4 . The calibrations are controlled by an automated calibration system capable of adapting to the varying conditions of each set pressure. This calibration system combined with the automated controls provides accurate reproducible calibrations over time. An example of the system stability is the zero pressure response voltage $V_{0}$ reproducibility shown in figure 3 . The variability of the 0.36 -psid ESP transducers voltage is $0.003 \mathrm{mV}$ over the 3 day test period, represented by runs 48 to 55 .

\section{Calibration Model Equations}

Voltage reproducibility over time illustrates the calibration system stability. However, calibration coefficients are the stability metric of interest. Two different calibration functional forms will be discussed. First, is the current regression model used by PSI in its System 8400. Equation 2 shows this regression model.

$$
p=C_{0}+C_{1} V+C_{2} V^{2}+C_{3} V^{3}+C_{4} V^{4},
$$

where $p$ is the pressure in psi, $V$ is the input voltage in Volts, and $C_{i}$ is the $\mathrm{i}^{t h}$ calibration coefficient, where $\mathrm{i}=0,1, \ldots 4$.

The second model proposed by Everhart ${ }^{6}$ is seen in equation 3 .

$$
p=A_{1} Z^{\frac{1}{3}}+A_{2} Z^{\frac{1}{2}}+A_{3} Z+A_{4} Z^{2},
$$

where

$$
Z=V_{i}-V_{0}
$$

Here, $p$ is the pressure, $A_{i}$, is the $\mathrm{i}^{\text {th }}$ coefficient where $\mathrm{i}=1,2, \ldots 4, V_{i}$ is the transducer output voltage, and $V_{0}$ is the offset voltage at zero pressure. Note that a direct comparison of the regression models can only be performed through an analysis of their curve fit residuals. This discussion will be addressed later in Section III.

\section{Environmental and Procedural Error Sources}

Several small experiments have been conducted that provide insight into the questions posed earlier in this paper. These questions must be answered to determine the controls required to expand the 15-psid transducer's usable range to include pressures down to 0.01 psia found in the hypersonic testing environment.

\section{A. System Noise}

What is the appropriate regression model to minimize calibration error? The appropriate regression for use in hypersonic wind tunnels is that which minimizes calibration curve fit error while providing stability and insensitivity to environmental error sources. In this analysis, a comparison of the PSI 4th order and Everhart regression models was conducted. However, before the comparison is conducted, a zero analysis was required to determine the noise band of the transducers.

The data from replicate calibrations taken over a two week time period from a single transducer were plotted in figure 4(a) and (c) on a linear scale and (b) and (d) on a semilog scale. With each replicate calibration, a corresponding measure of the zero pressure voltage $V_{0}$ was acquired. These voltage measures, which can be seen best in figure 4(b) of the 0.36-psid ESP data and figure 4(d) of the 15-psid ESP scattering data around $P=10^{-5}$, have no correlation with the pressure settings at this level. This is indicative of the noise floor of the measurement system; thus the zero pressure voltage dispersion measure can be used to quantify the instrument's noise band. Replication of measures at pressure can also yield this information; 
however, care must be taken in the data acquisition such that there is little or no set point error. This can cause a local correlation effect and bias the noise measure.

The ranges from each of eight randomly selected ports on a given module are compared for consistency. This is done to estimate an average noise band for a given module range. Figures 5 (a) and (c) show a SPC range charts for the 0.36 and 15 psid ESP modules, respectively, and figures 5 (b) and (d) present the same results in terms of percent of full scale ( 5 volts). Calculations for figure 5 were based on basic SPC theory outlined by Wheeler and Chambers, ${ }^{2}$ where the constants were taken from Tables A.1 and A.2 on pages 393-394.

Based on the results from figure 5, each port has essentially the same variation and the average variation across the ports is assumed to be indicative of the behavior of a transducer for that range of gage. The standard deviation can be estimated by $\hat{\sigma}=\bar{R} / d_{2}$. For $n=10, d_{2}=3.078$. The noise of the instrument will be represented by the standard error band $\pm 3 \hat{\sigma}$. Thus the instrument noise can be expressed as

$$
\pm 3 \hat{\sigma}= \pm 3 \frac{\bar{R}}{d_{2}}= \pm \frac{3 \bar{R}}{3.078}= \pm 0.975 \bar{R} \approx \pm \bar{R} .
$$

Based on the acquired data, the 0.36-psid and 15-psid transducers have a noise band of $\pm 4.1 \mathrm{mV}$ or $\pm 0.082 \%$ full scale and $\pm 1.2 \mathrm{mV}$ or $\pm 0.023 \%$ full scale, respectively. Due to the project goal of extending the usable range of the 15-psid transducers to lower pressures, the 0.36-psid transducer will only be discussed to illustrate the effects of over-pressurization. Therefore, the two regression models are compared based on the 15-psid transducer.

\section{B. Regression Model}

System $8400^{1}$ transducers are normally calibrated using the intrinsic calibration algorithm that sets, measures, and fits a fourth order curve to 5 data points. This built-in system does not provide sufficient degrees of freedom to enable error testing for the calibration curve as highlighted in Everhart. ${ }^{6}$ Without extra degrees of freedom for curve fit error detection, the calibration curve must pass through each data point, providing no error at those points and possibly accentuating error in between. Adding degrees of freedom, as done within these experiments, provides a conservative analysis of the 4th order regression model and an equal footing for both regression models to be compared.

For the purpose of comparison, equation 2 will be modified, without a loss of generality, to incorporate the same shifted $\mathrm{Z}$ voltage used by Everhart and shown in equation 4. The resulting Regression model is shown below in equation 6 .

$$
P=B_{1} Z+B_{2} Z^{2}+B_{3} Z^{3}+B_{4} Z^{4}
$$

Figure 6 illustrates the curve fit of the 4th order (a) and Everhart (b) regression models for two separate cases. First is the curve fit from calibrations using 32 degrees of freedom in subfigure (a) and (b), where the second set of curve fits (subfigures (c) and (d)) were generated using only 9 degrees of freedom. These plots show the curve fits on a $\log$ scale of pressure versus the $\log$ of the shifted $\mathrm{Z}$ voltage. Figure 6 , the full scale curve fits, have four elements: the data used for the regression process, the curve fit result from the regression process, the prediction interval of an individual measurement using a significance level of $\alpha=0.05$, and the cross-check data consisting of data from the 9 other calibration runs.

Subfigures (a) and (c) illustrate that the 4th order curve fit does not follow the trend of the data at low pressures, while the Everhart regression model curve fit shown in subfigures (b) and (d) follows the data more closely. The insensitivity of the 4 th order curve fit is due to the lack of low pressure nonlinearity terms, which Everhart introduced as $Z^{\frac{1}{3}}$ and $Z^{\frac{1}{2}}$.

The cross-check data will also be used to generate predicted residuals by subtracting the actual measure of pressure from the Ruska standard from the predicted value of pressure from the curve fit. This process of predicting the residual is accomplished by taking an ordered pair of measured data, $\left(V_{i}, P_{i}\right)$, and transforming it to $\left(V_{i}, \dot{r}_{i}\right)$ by using the following equation:

$$
\dot{r}_{i}=\hat{P}_{i}\left(V_{i}\right)-P_{i},
$$

where $\dot{r}_{i}$ is the individual predicted residual. The subfigures in figure 7 plot the residuals of the data versus the $\log$ of the $\mathrm{Z}$ voltage, compare the magnitude of the Everhart and 4th order regression model Data and predicted residuals for the 32 and 9 degree of freedom cases. Subfigures (a) and (b) show that the Everhart 
model residuals vary randomly about zero, where the 4th order residuals (subfigure (c) and (d)) show a significant error to one side or the other based on the pressure. When data and predicted residuals all fall to one side of zero, it illustrates the inability of the regression model to fit the data in these regions.

The absolute residual shown in figure 8 (a) through (d) show the absolute values as a percentage of full scale versus the $\log$ of the $\mathrm{Z}$ voltage. They illustrate how much better the Everhart regression model fits the data relative to the 4 th order curve fit. The 4 th order model shown in figure 8(a) and (c) have prediction limits outside the noise bands. The prediction interval using Everhart's model shown in figure 8(b) and (d) are within the noise band of the transducers. ${ }^{\mathrm{b}}$

Figure 8(b) and (d) illustrates the prediction interval of the Everhart Model based on 32 and 9 calibration points, respectively. The prediction interval for this model changes very little as degrees of freedom are decreased from 27 to 4 degrees of freedom. However, reducing the degrees of freedom from 27 to 4 increases the prediction interval for the 4 th order regression model by $33 \%$, as shown in contrast between figure 8 (a) and (c). The 4th order model does not simulate the physical nature of the response which caused the prediction interval to increase; whereas Everhart's model is very similar. This fact is illustrated in Everhart ${ }^{6}$ by the 4th order regression model's point placement sensitivity.

Five degrees of freedom are required to determine calibration coefficients of both the 4 th order and Everhart's regression models due to the number of coefficients being estimated. Six degrees of freedom is sufficient to test for curve fit error, enabling the Everhart model residuals fall within the noise band of the ESP transducer. Figure 8(a) and (c) illustrates how the 4th order regression model fails to perform as well as the Everhart model even with 32 degrees of freedom. Due to the substantial increase in measurement uncertainty generated by the 4th order regression model, it will not be discussed further with the exception of the 4th order regression model's point placement sensitivity found in Section III-D.

\section{Temperature Effects}

How sensitive are the ESP transducers to temperature fluctuations? Are the calibration coefficients sensitive to temperature change? These questions can be answered by the individuals and moving range charts shown in figure 9 . Note that there are actually 6 separate, 4-point, individuals and moving range charts in these figures, one per temperature setting denoted above. These charts illustrate the mean value and variability of the offset voltage and calibration coefficients of the Everhart regression models relative to each temperature setting. The temperature set points are: $95.0,95.25,95.5,96.0,97.5$, and $100.0^{\circ} \mathrm{F}$ as noted above the data in the figures. Temperatures are controlled to $\pm 0.1^{\circ} \mathrm{F}$ as discussed in Green. ${ }^{3}$ Figure 9 shows that the 4-point data sets, in general, vary randomly about the process mean and are in statistical control.

The Everhart regression model described in equation 3 and shown in figure 9 (a) has five calibration coefficients, one being the zero pressure voltage $\left(V_{0}\right)$ used to calculate $\mathrm{Z}$ in equation 4 . This constant term $V_{0}$ is sensitive to temperature fluctuations exceeding $0.25^{\circ} \mathrm{F}$ as shown in figure 9 (b) which contains repeated calibrations of a 15-psid transducer. Significance of the temperature response fluctuation is based upon the natural process limits calculated using the SPC process and shown as UCL and LCL as indicated in the legend in figure $9(\mathrm{a})$. Temperature changes do not effect the mean values of the other 15-psid transducer coefficients presented in the individuals charts of the (c) to (f) subfigures in figure 9 until temperature has changed more than $2.5^{\circ} \mathrm{F}$.

The mean values of the calibration coefficients taken at each temperature setting reside within the control limits of all the other temperature settings until this point. Variation of the coefficients seems to change with module temperature. However, the majority of the variation present at each temperature is likely not significantly different than variation found in at the other temperatures. Because the coefficient values vary in a controlled manner around the mean value, the question arises, should the mean coefficient values be used for pressure calculation rather than the last calibration coefficients? If the mean coefficient value is used, added variation based on insignificant temperature variation during transducer calibration will not increase pressure measurement uncertainty. Therefore, the mean coefficient values can be used until the temperature control system no longer acts in a statistically controlled manner. ${ }^{\mathrm{c}}$

\footnotetext{
${ }^{\mathrm{b}} \mathrm{A}$ notable exception here is that the prediction interval is greater than the instrument noise band beyond where the data is. This is because the calibration is not valid in the extrapolated region.

${ }^{\mathrm{c}}$ The 0.36 -psid transducer is slightly more sensitive to temperature shifts, which is most likely caused by different material properties of the smaller range transducer.
} 


\section{Environmental Pressure}

Does the transducer calibration change when the environmental pressure changes? Figure 10 shows that changes in the environmental pressure in which the ESP modules reside significantly affect the 15-psid transducer $V_{0}$ voltages and calibration coefficients regressed for the Everhart model. These figures were generated using regressed calibrations conducted in run order. The first seven runs were conducted with the ECC evacuated, then pressurized to atmosphere for the next seven runs. The Everhart regression coefficients seen in figure 10, with the exception of $V_{0}$, do not have a significant mean shift. However, the variation of the coefficients increases significantly. Therefore, both the mean value $V_{0}$ and variation of the $A_{i}$ coefficients are significantly affected by the environemental pressure. These figures show that measurements and calibrations must be acquired under the same environmental conditions due to leaks found between the environment and reference chamber as well as structural strain effects applied to the transducers. ${ }^{\mathrm{d}}$

A side note, the effect of environmental pressure on the 4th order curve coefficients are shown in figure 11. Subfigures (c), (e), and (f) show a point outside the control limits corresponding to Run \#1. During an analysis of the calibration data for this run, it was found that the last two calibration points were taken at pressures other than normally set due to a problem with the control algorithm programming. Therefore, the last two data points were removed from the run and the remaining points were regressed. In contrast to the Everhart coefficients shown in Figure 10 where no effect was seen, the 4th order calibration shows significant changes in the coefficients. The coefficients regressed from pressure and voltage data should not be directly dependent on the magnitude of the set calibration pressures. Everhart ${ }^{6}$ discussed this issue when he showed that the 4 th order regression model produced different calibration curves based on the placement of the calibration set points. It is not recommended that a calibration curve be used past the last calibration point, due to the flaring of the prediction interval beyond the last data point as seen in figure 6(a) and (b). Although, it is very comforting that the Everhart coefficients are insensitive to the placement of the calibration points to this degree.

\section{E. Randomization Effects}

Randomization is a tool used to ensure independence which is required for the use of formal experimental design methods. However, is randomization of set points required to improve transducer calibration accuracy? To answer this question, a study was conducted where four calibrations were performed with non-randomized set points and four runs were conducted with randomized set points. Non-randomized calibrations start with the entire system at hard vacuum. Higher set pressures are applied in order of increasing magnitude so that out-gassing is not required to fully settle the pressure. Randomized set point values were generated using a software randomization algorithm for each run. The randomized set points were then used to calibrate the transducers without extra time provided for out gassing.

This study showed how set point randomization, when no extra time was afforded for out-gassing, significantly affects the 15-psid ESP transducer response voltages and regressed coefficients of the Everhart regression model as seen in figure 12. Residual gas pressure also causes the mean voltage measurement values to shift as seen in figure 12(b). The mean values of calibration coefficients $A_{1}$ and $A_{2}$ seen in figure 12(c) and (d) shift significantly from non-zero to values not significantly different from zero, when randomizing the calibration runs in this manner. Therefore, properly out-gassing the calibration system is required for randomization. However, providing the required out-gassing at each set point is time prohibitive, when low pressure points are set after high pressure. At low pressure conditions, significant time is required for the calibration system to equilibrate resulting in direct operational and facility productivity issues.

\section{F. Effects of Over-Pressurization}

Does over-pressurization of an ESP transducer require a new calibration? The answer to this question is illustrated in figure 13 which depicts the history of the calibration system detailed fully in Green. ${ }^{4}$ During the tuning process where system control settings were specified, the calibration system allowed pressures to over-shoot the set pressures. Many times the over-shoot exceeded $400 \%$ of the 0.36 -psid transducers pressure range. This region is noted above those points in the individuals chart of figure 13. As the systems tuning was improved, the over-pressurization deceased below $200 \%$ of range as illustrated by a trend line shift to

\footnotetext{
${ }^{\mathrm{d}}$ The 0.36 -psid transducer was similarly affected by the environmental pressure change. Although, the mean value of $V_{0}$ shifts greatly.
} 
a shallower gradient. At run 42, overshoot was eliminated which allowed the $V_{0}$ to stabilize within control limits calculated from the stabilized voltage measurements.

In contrast, figure 14 depicts the $V_{0}$ voltages from the 15-psid transducer. Even with all the adjustments made to the calibration system to eliminate the 0.36-psid over-pressurization, the 15-psid transducer $V_{0}$ measurements remained in statistical control. The 15-psid transducer is incapable of being over-pressurized under these operational conditions, gradually fell into a random behavior about the mean $V_{0}$ voltage, as shown in figure 14 . Therefore, over-pressurization has an adverse effect on the 0.36 -psid transducer calibration reproducibility over time.

Figure 15 shows that regression coefficients for the 0.36 -psid transducer full optimization process which in general are in statistical control for the Everhart regression model. Though the calibration system was not yet fully stable, the coefficients $\left(A_{i}\right)$ did not show any trends similar to those seen on the $V_{0}$ individuals charts. This illustrates that over-pressurization should be detected by a shift in the zero pressure voltage data. Once a shift is detected, a full re-calibration should be conducted to determine if the other regression coefficients have been affected, however unlikely. The same trends are seen in runs 1 through 55 in figure 16 for the 15-psid transducer. More research is necessary to determine the full effects of transducer over-pressurization on regression coefficients and the amount of over-pressurization that a transducer can withstand without shifting zero pressure voltage responses.

\section{G. Calibration Interval}

What should the re-calibration time interval be? The 15-psid transducer is very stable over time. Figure 16(b) is an SPC plot of the $V_{0}$ values obtained over the duration of testing at relatively fixed conditions when the system was functioning properly. Note the behavior seen on the full individuals plot. $V_{0}$ appears to drift over the full $2.5 \mathrm{mV}$ span over the 3 months of calibrations conducted. Recall, the first 55 data points were obtained when numerous configuration changes occurred. This $2.5 \mathrm{mV}$ contains the System 8400 s noise floor and long term drift, in addition to the calibration systems long term drift.

Examining the rest of figure 16, the low-pressure coefficients tend to drift in the same manner as $V_{0}$, where high pressure coefficients vary randomly around their means. The low pressure coefficients seem to have some dependence on the offset voltage and vary similarly with the system long term drift. Therefore, mean calibration coefficients obtained under short term conditions can be used, so long as $V_{0}$ remains stable within the $\pm 1.2 \mathrm{mV}$ span between the control limits. Once the offset voltage drifts out of control, a full calibration must be conducted to verify the integrity of the calibration coefficients.

Using the mean coefficient values minimizes induced variation due to frequent re-calibrations and makes the utilized coefficients less sensitive to small environmental changes, as discussed previously. In this case, where the regression model is fitting the calibration curve, minimizing residuals, to a level within the system noise band, re-calibrating early only shifts the calibration within the noise floor of the system and provides no significant benefit.

\section{H. 15-psid Calibration Extent}

Can the 15-psid transducer be calibrated to measure pressures down to 0.01 psia? Because the calibration can only be as good as the instrument, this question can be answered looking at the noise band of the 15-psid transducer. The noise level described previously in Section III-A shows that measurement uncertainty of the 15-psid transducers tested can be decreased by half. This result allows the 15-psid transducer to be calibrated with residual values that fall within the transducer noise band which is approximately $0.023 \%$ of full scale. This calibration, with the environmental controls applied, allows measurement accuracy to within \pm 0.0035 psia which is well below the free-stream static pressures of many hypersonic wind tunnels.

Except for special testing conditions, the 15-psid transducer can be used without the problems associated with over-pressurization to make accurate measurements, over time, without full re-calibrations, given that the offset voltage remains stable. However, this test and analysis has characterized only 8 randomly selected transducers on one 15-psid module. A zero pressure analysis is required on all transducers used in this manner to determine if they have the same abilities as found here. More testing is necessary to determine if these properties can be applied generally to other 15-psid modules. 


\section{Conclusions}

The 15-psid ESP transducer has the capability to be used accurately in the hypersonic environment when calibrated using appropriate regression methods and proper environmental controls are provided. Using the Everhart regression model allows the transducer to be calibrated to the error band of the transducer. This model captures the natural behavior of the transducers when used as absolute gages.

The reported uncertainty of the transducer can be reduced by a factor of 2 when proper temperature and environmental pressure controls are provided. The required environmental sensitivity of these transducers has been studied providing the following guidance relative to their use in hypersonic wind tunnels where in-situ calibrations cannot be conducted.

Small temperature changes significantly affect the mean value of the 15-psid ESP transducer response voltages, when temperature changes are greater than $0.5^{\circ} \mathrm{F}$. To minimize measurement error due to temperature, the mean calibration coefficients determined from repeated calibrations should be used. The mean coefficient values provide calibration stability because the calibration is sensitive to the noise band of the transducers.

Changes in the environmental pressure surrounding the ESP module significantly affect the calibration of ESP transducers by increasing the variance of the zero pressure voltage and calibration. Therefore, the environmental pressure must remain constant between calibration and test measurements to ensure valid measurements, particularly at low pressures.

Proper out-gassing at randomized set pressures points is required. Otherwise, insufficient out-gassing adversely affects low-pressure calibration of ESP transducers. Alternatively, calibrating in order of ascending pressure allows calibrations to be conducted within an acceptable time frame and with residuals that fall within the noise band of the transducers because pressure equilibrium is maintained within the calibration system.

Over-pressurization significantly affects the accuracy of the 0.36 -psid transducers by shifting the mean offset voltage. With environmental controls in place, over-pressurization can be minimized in use but may occur when 0.36-psid transducers are used as an absolute gage. However, the 15-psid transducers cannot be over-pressurized using this calibration system or under normal hypersonic wind tunnel operating conditions.

The testing of powered pressure models, where surface pressures may change by two orders of magnitude, requires the use of 15-psid transducer. Extending the usable range of the 15-psid transducer to low pressures is possible when environmental controls are provided by an automated calibration system with tightly defined control algorithm coupled with a regression method (Everhart) that provides residuals smaller than the system noise. The 15-psid transducers studied can be calibrated to an accuracy of $0.023 \%$ FS which allows pressures to be measured within \pm 0.0035 psia.

Monitoring the ESP Calibrations using Statistical Process Control methods is critical in maintaining accurate pressure measurements. The zero-pressure voltage is the key indicator of the health of the transducer. Analyzing the zero-pressure voltage values prior to each test run provides the ability to spot check the system. Another method of maintaining calibration accuracy is to use the mean calibration coefficient values. The use of mean values will minimize coefficient variation due to system noise. Once significant drift in the zero offset voltage is detected, a full calibration should be conducted to verify the coefficient values due to the zero pressure voltage being sensitive to environmental conditions. Using this calibration process can enable full calibrations to be taken at intervals exceeding 2 weeks making facilities more productive.

Future testing should involve repeating these studies on other 15-psid modules determining if other transducers have the same capability as these tested. Other environmental error sources such as vibration should also be evaluated so as to provide limits and mitigations required to maintain calibration and measurement accuracy.

\section{Acknowledgments}

The author would like to thank Anthony W. Robbins of Gas, Fluid, and Acoustic Research Support Branch, Research \& Technology Directorate, NASA Langley Research Center) for his technical knowledge of instrumentation systems used in hypersonic wind tunnels and for his enthusiasm and expertise in constructing the high vacuum constrained calibration control system. 


\section{References}

${ }^{1}$ Pressure Systems Inc. System 8400 Users Manual, 1999.

${ }^{2}$ Donald J. Wheeler and David S. Chambers. Understanding Statistical Process Control. SPC Press, 2nd edition, 1992.

${ }^{3}$ Del L. Green, Matthew N. Rhode, and Joel L. Everhart. Development and Characterization of a Low-Pressure Calibration System for Hypersonic Wind Tunnels. AIAA Paper 04-0600, AIAA, January 2004.

${ }^{4}$ Del Lloyd Green. Statistical Characterization of a Pressure Calibration System Developed for Low Pressure Transducer Calibrations. Master's thesis, The George Washington University, 2005.

${ }^{5}$ Donald J. Wheeler and Richard W. Lyday. Evaluating the Measurement Process. SPC Press, 2nd edition, 1989.

${ }^{6}$ Joel L. Everhart. Calibration Improvements to Electronically-Scanned Pressure Systems and Preliminary Statistical Assessment. AIAA Paper 96-2217, AIAA, June 1996.

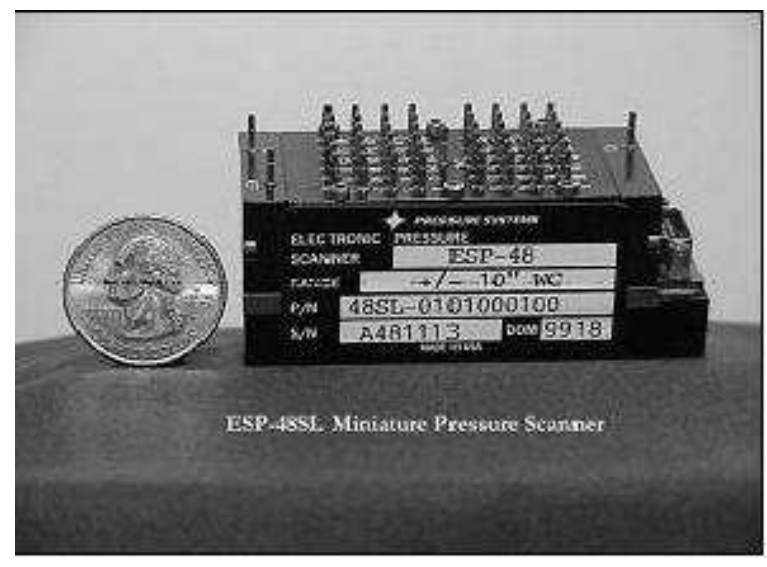

Figure 1. ESP Transducer Module
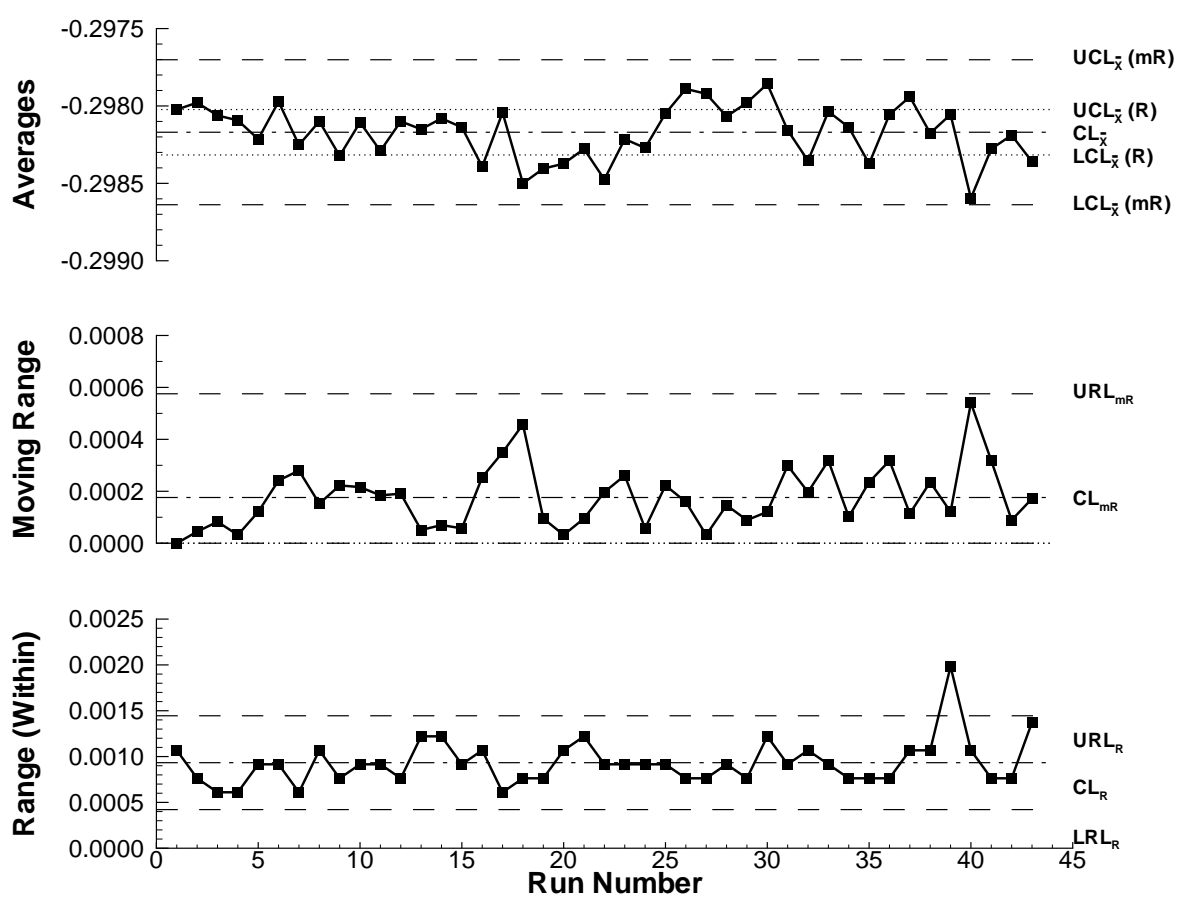

Figure 2. Example $V_{0}$ Voltage Reproducibility Chart 

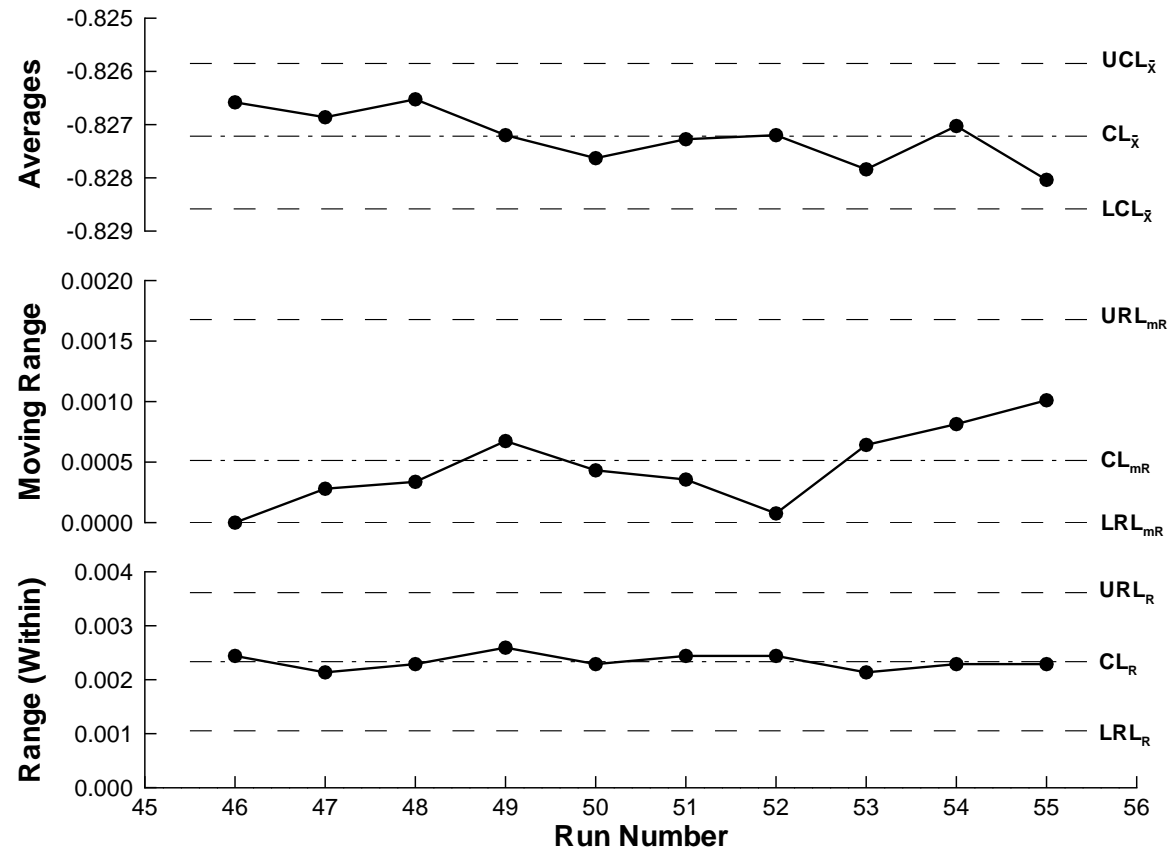

Figure 3. Zero Pressure Voltage Reproducibility 


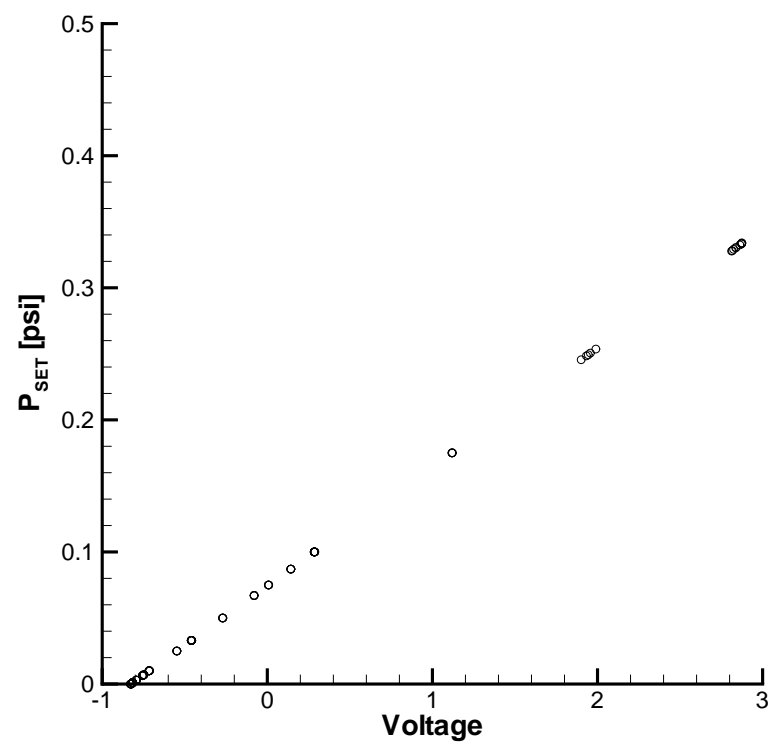

(a) Linear Scale (0.36 psid)

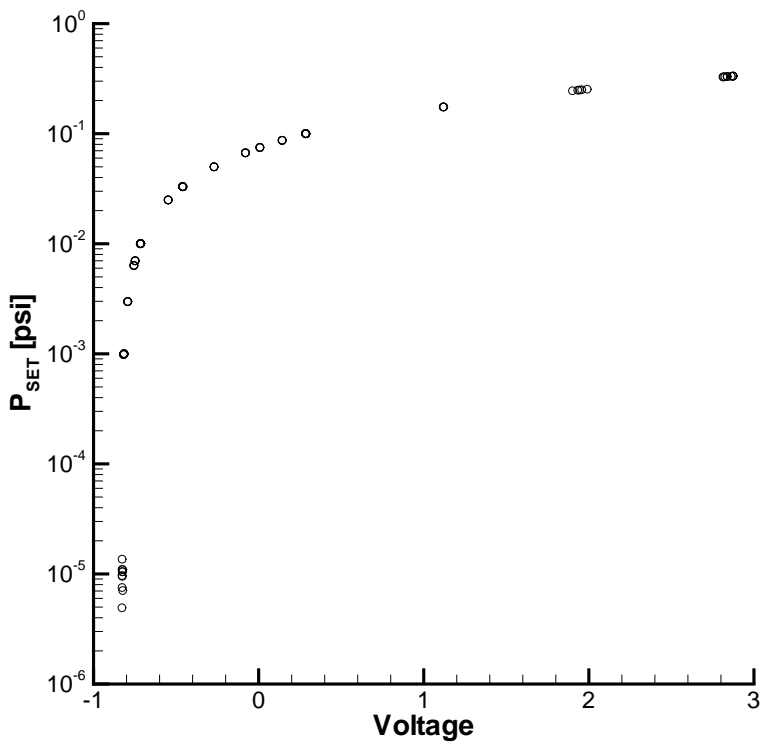

(b) Semilog Scale (0.36 psid)

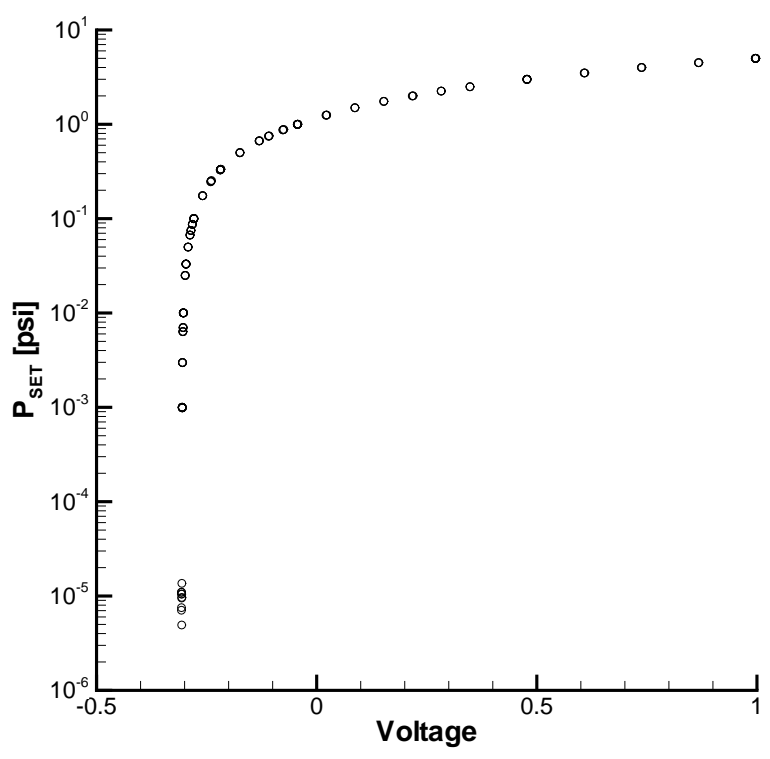

(d) Semilog Scale (15 psid)

(c) Linear Scale (15 psid)

Figure 4. ESP Calibration Data (Note change of scale.) 


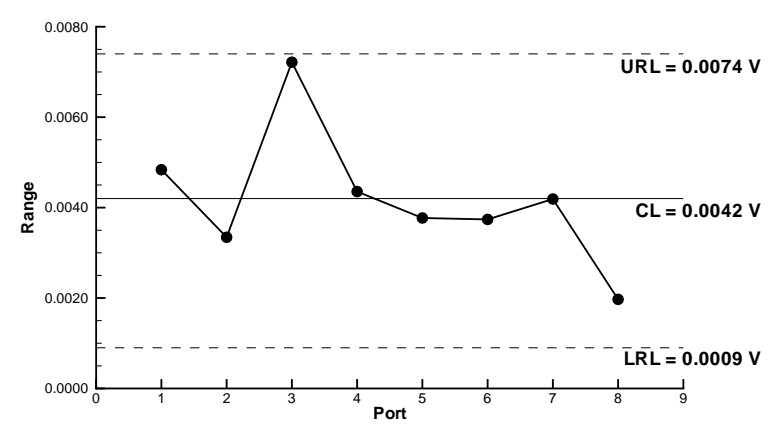

(a) Voltage (0.36 psid)

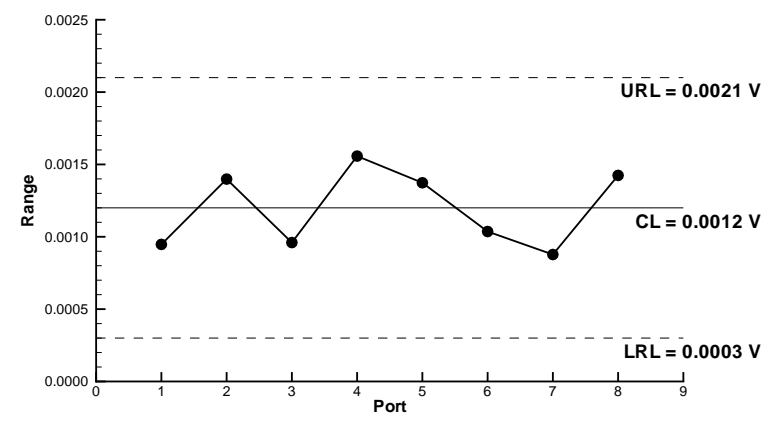

(c) Voltage (15 psid)

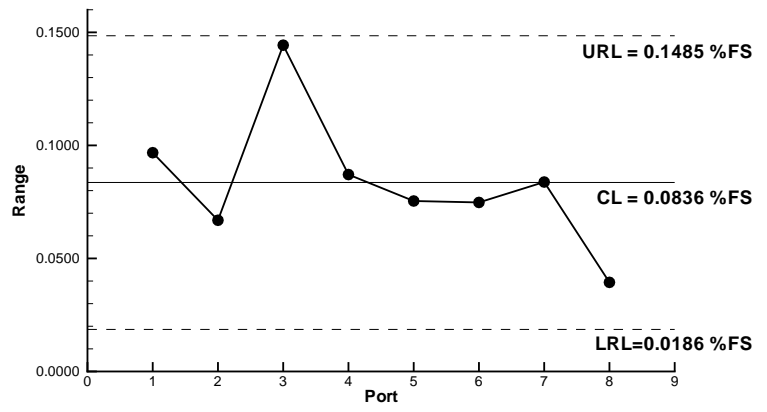

(b) \% Full Scale (0.36 psid)

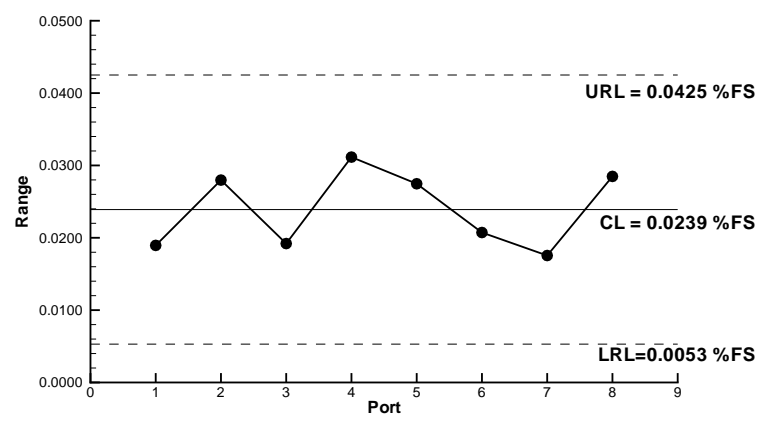

(d) \% Full Scale (15 psid)

Figure 5. Zero Voltage Range Analysis 


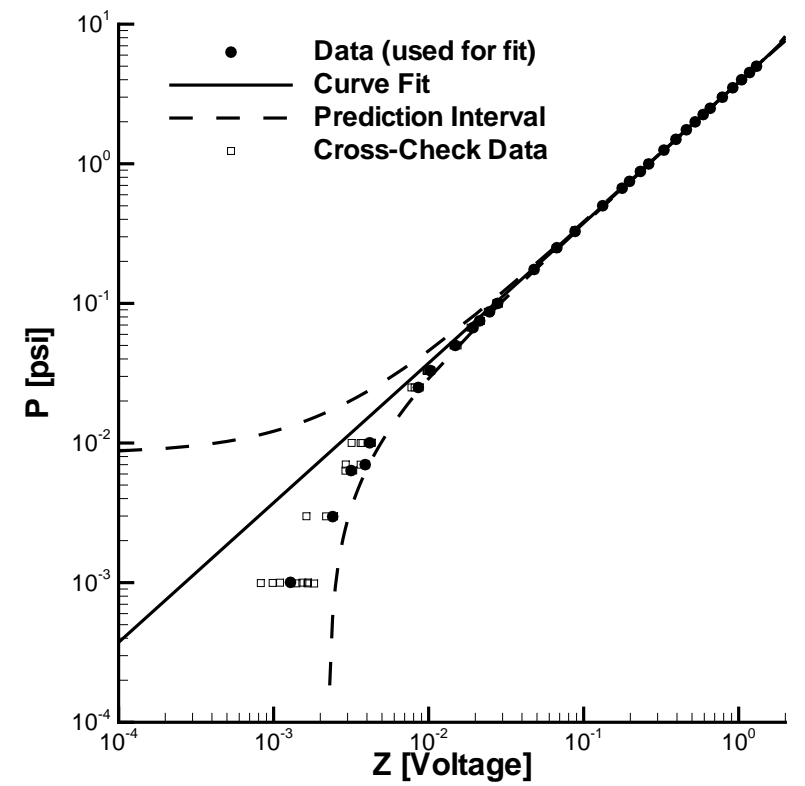

(a) 4th Order Polynomial / 32 point Calibration

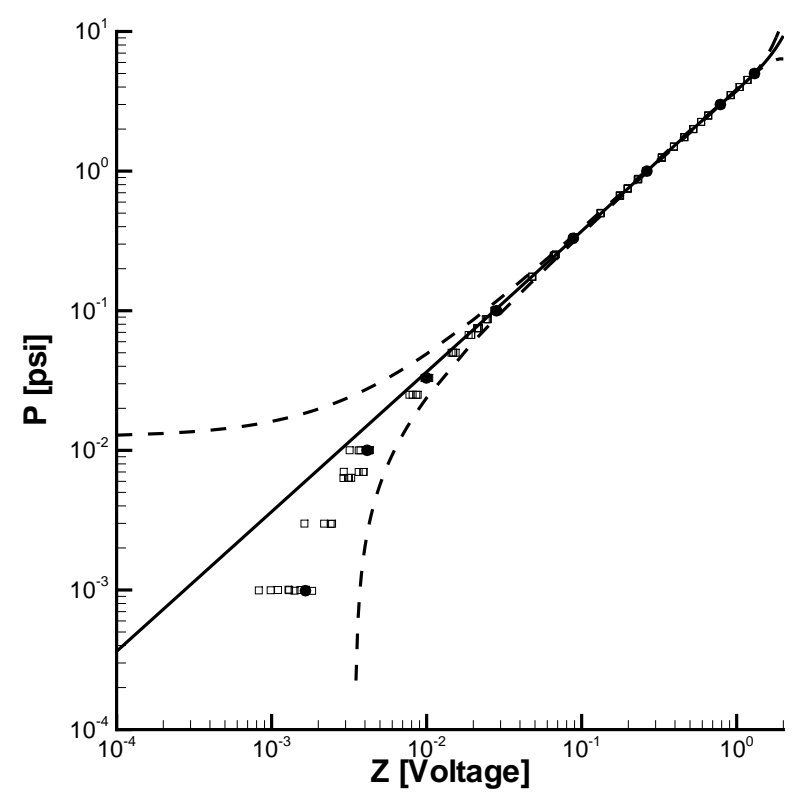

(c) 4th Order Polynomial / 9 point Calibration

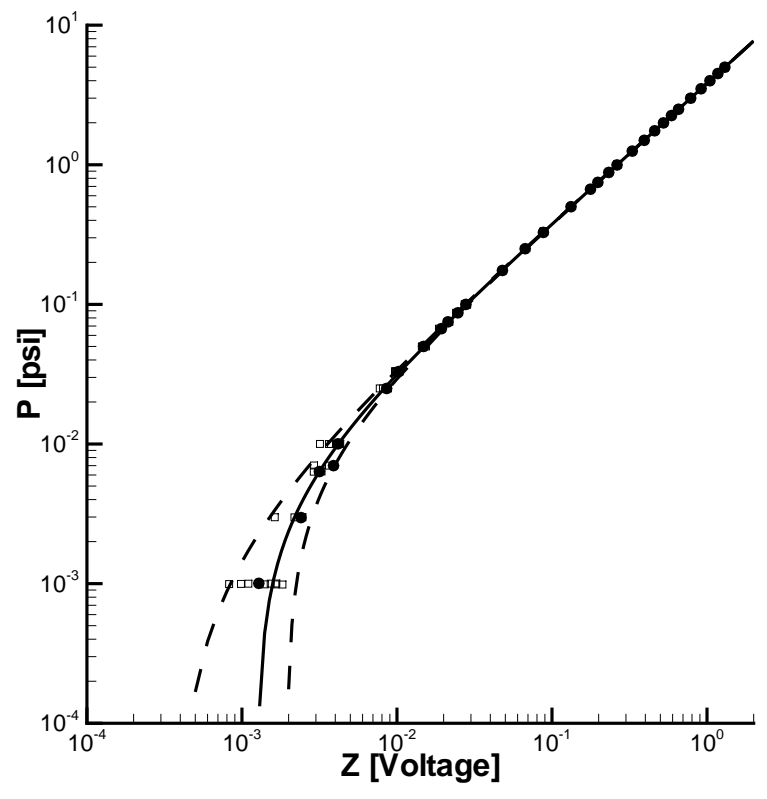

(b) Everhart Model / 32 point Calibration

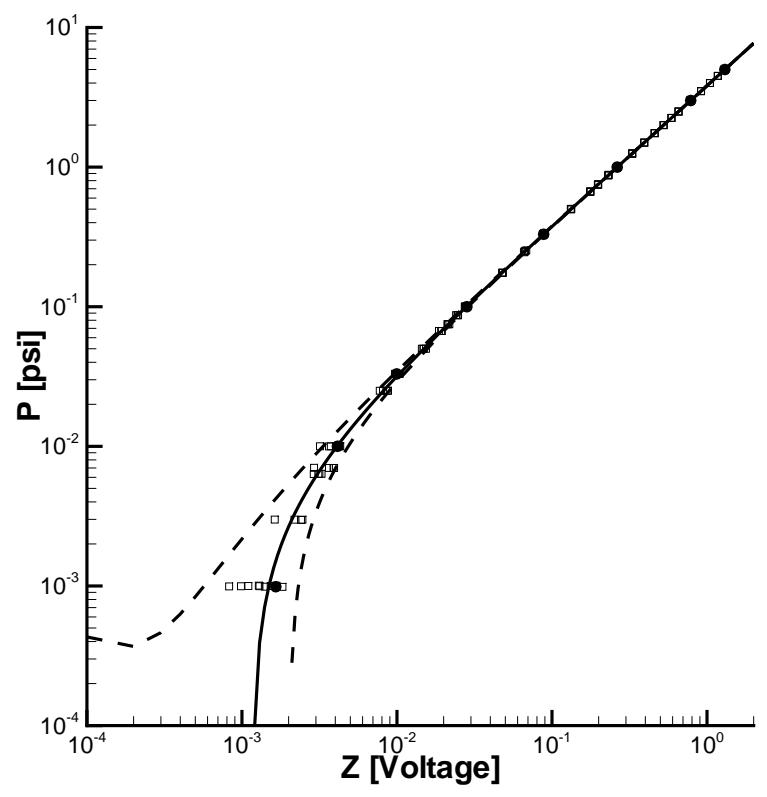

(d) Everhart Model / 9 point Calibration

Figure 6. Curve Fit Comparison for 15-psid ESP 


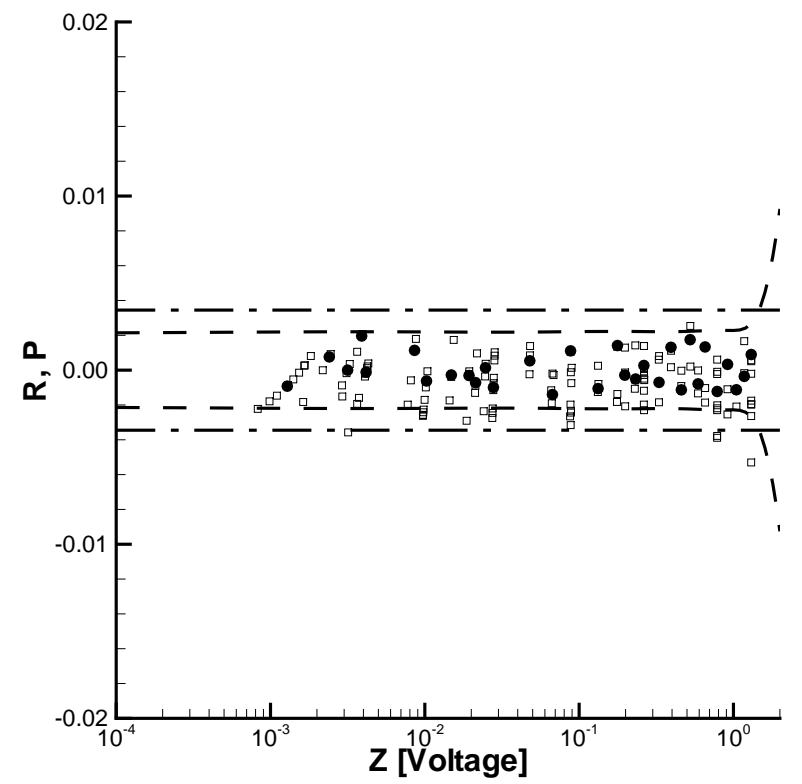

(a) Everhart Model / 32 point Calibration

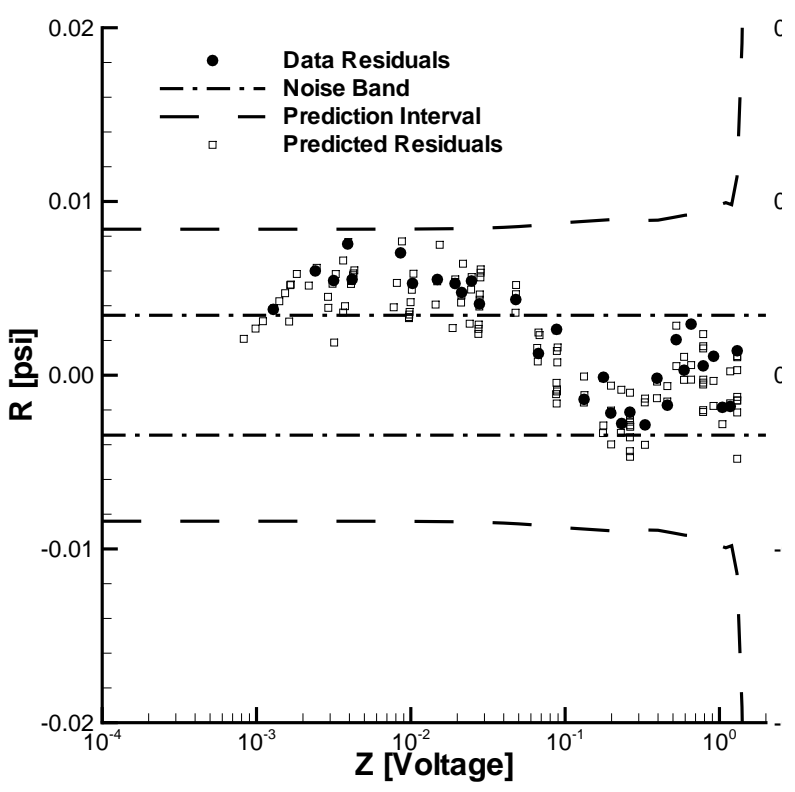

(c) 4th Order Polynomial / 32 point Calibration

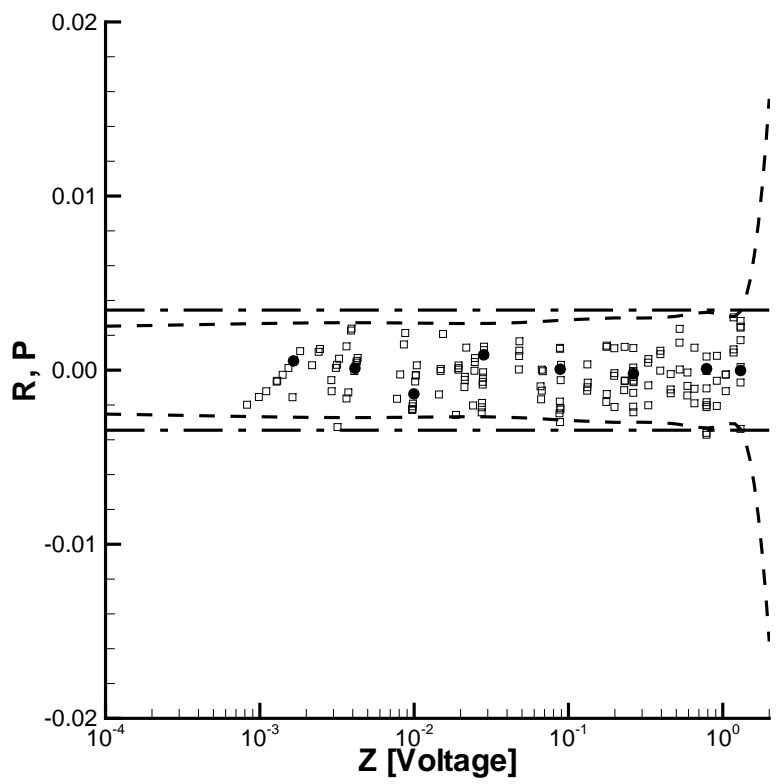

(b) Everhart Model / 9 point Calibration

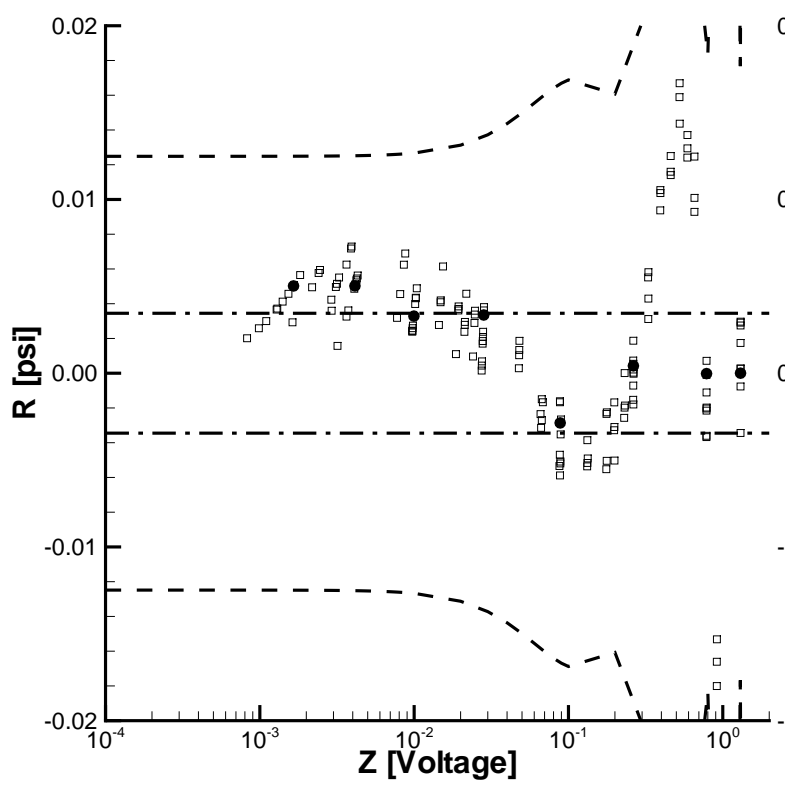

(d) 4th Order Polynomial / 9 point Calibration

Figure 7. Residual Comparison for 15-psid ESP 


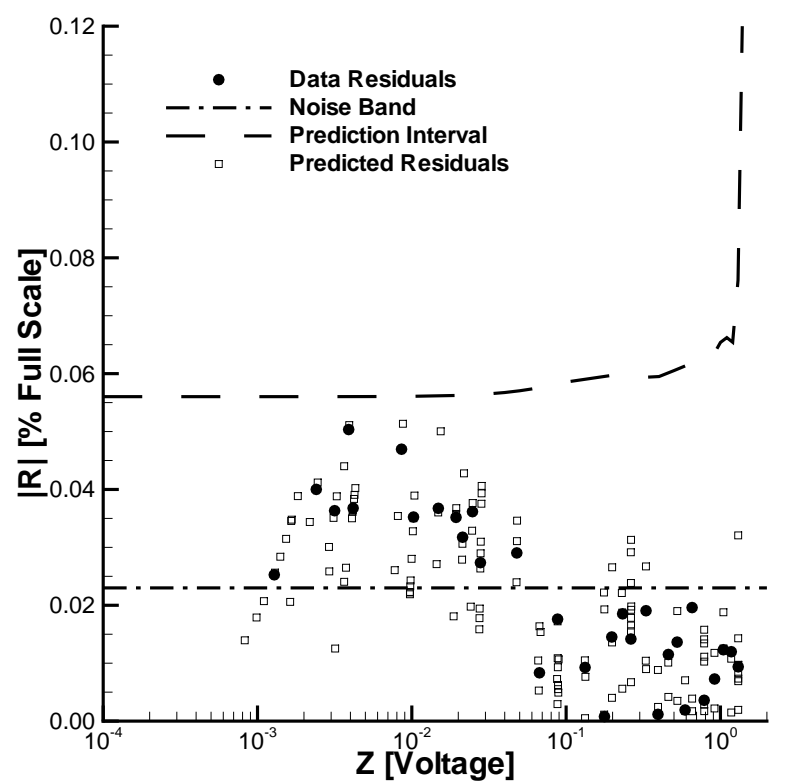

(a) 4th Order Polynomial / 32 point Calibration

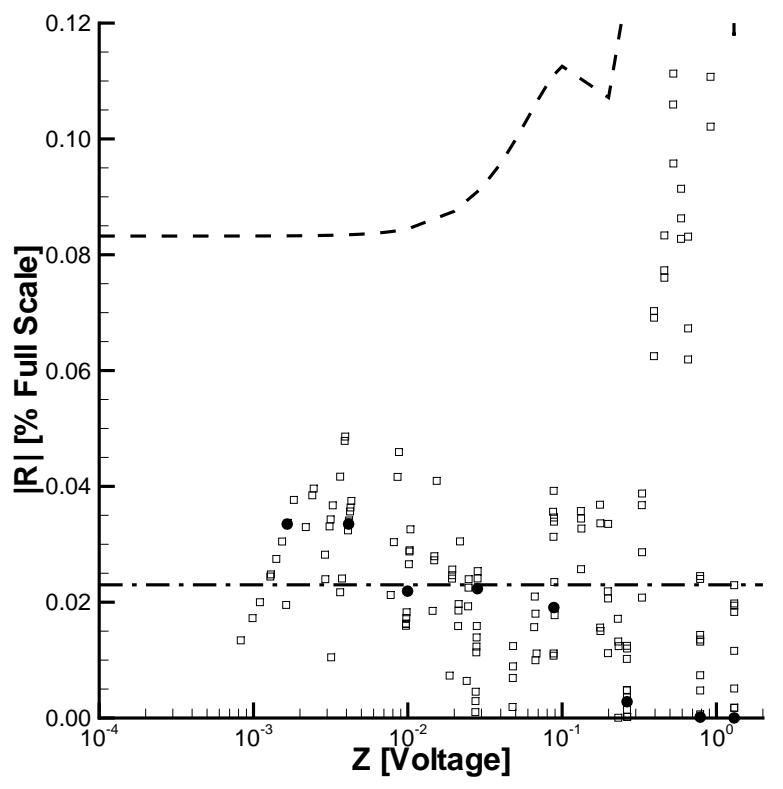

(c) 4th Order Polynomial / 9 point Calibration

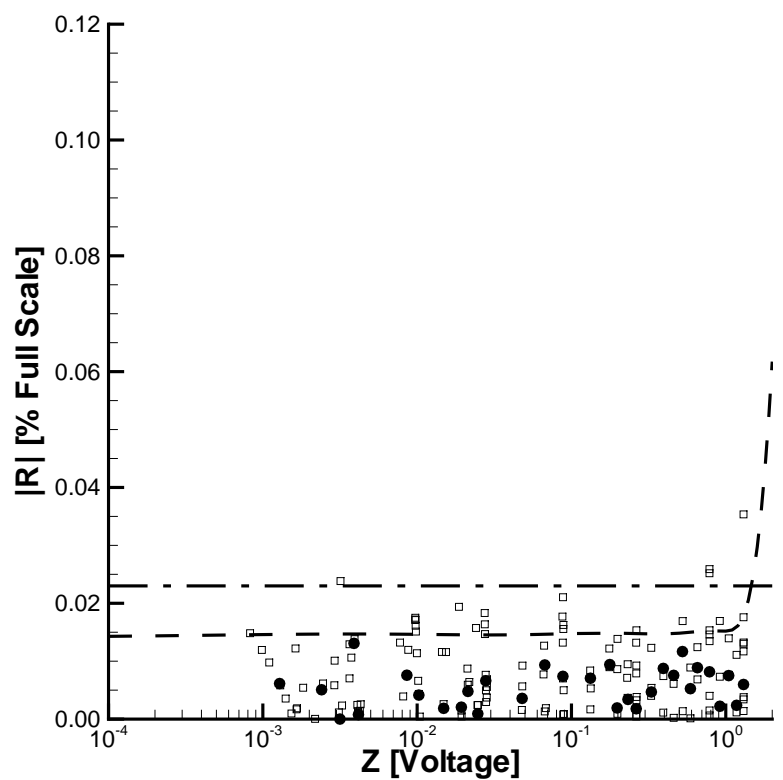

(b) Everhart Model / 32 point Calibration

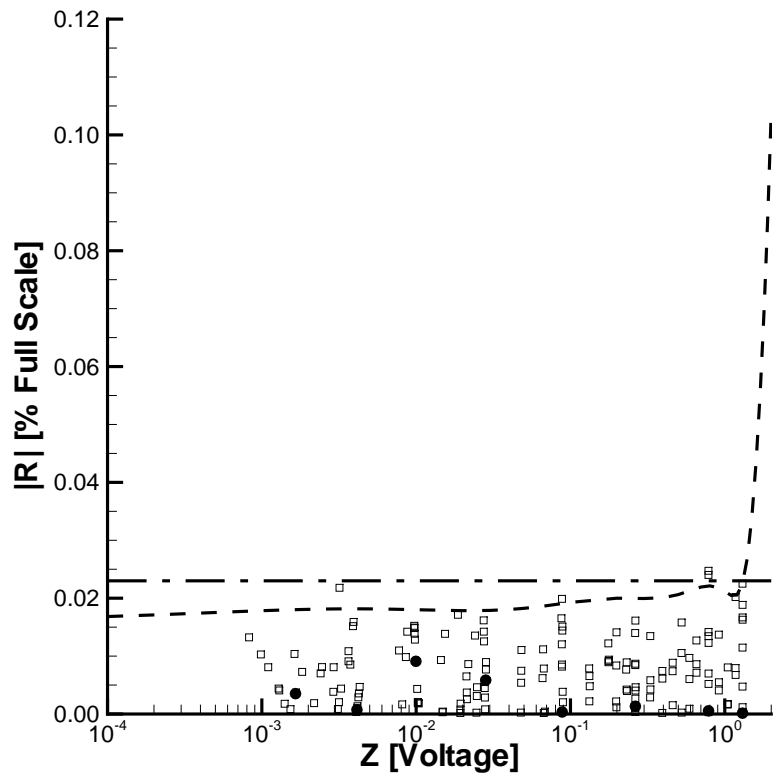

(d) Everhart Model / 9 point Calibration

Figure 8. Absolute Residual Comparison for 15-psid ESP in \% of Full Scale 
$p=A_{1} Z^{\frac{1}{3}}+A_{2} Z^{\frac{1}{2}}+A_{3} Z+A_{4} Z^{2}$

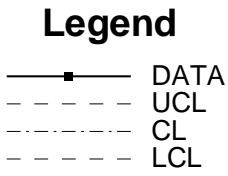

(a) Functional Form and Legend

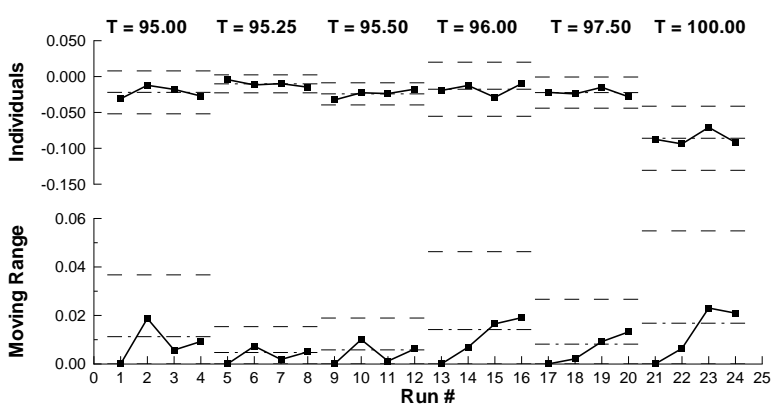

(c) $A_{1}$ Coefficient
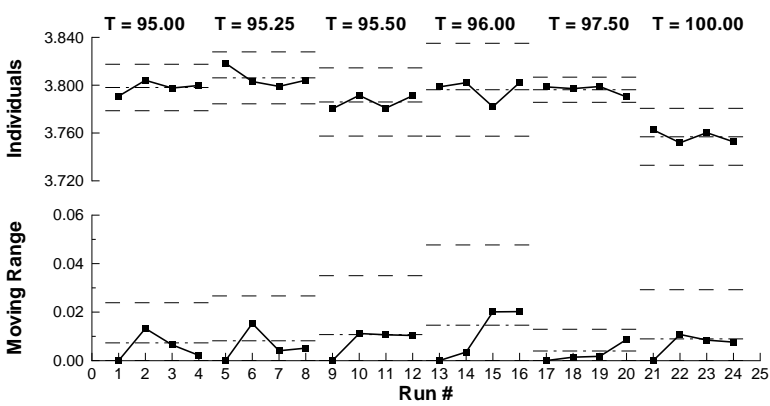

(e) $A_{3}$ Coefficient
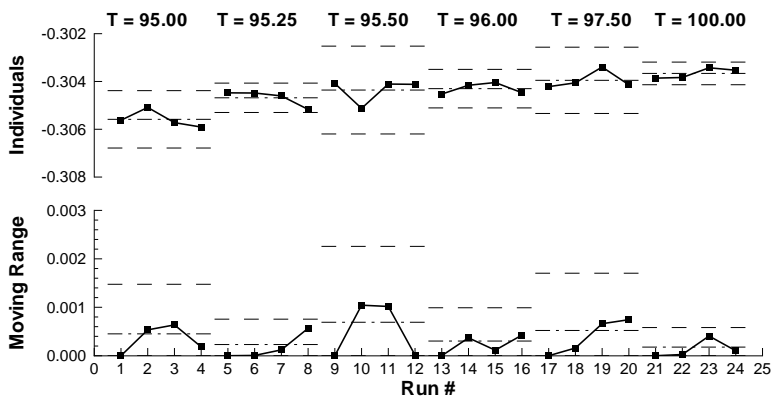

(b) $V_{0}$ Coefficient
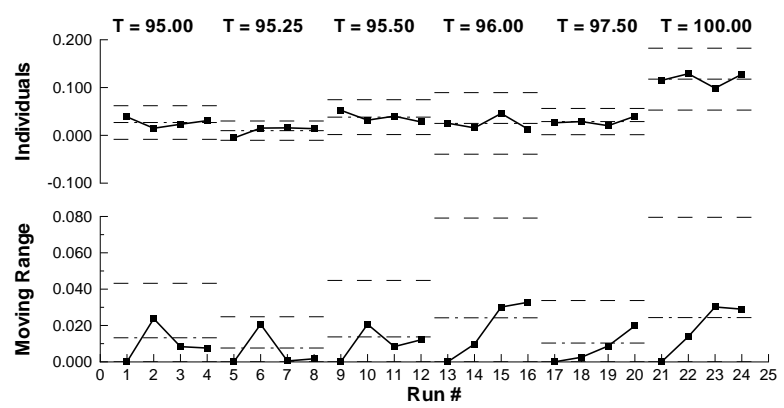

(d) $A_{2}$ Coefficient
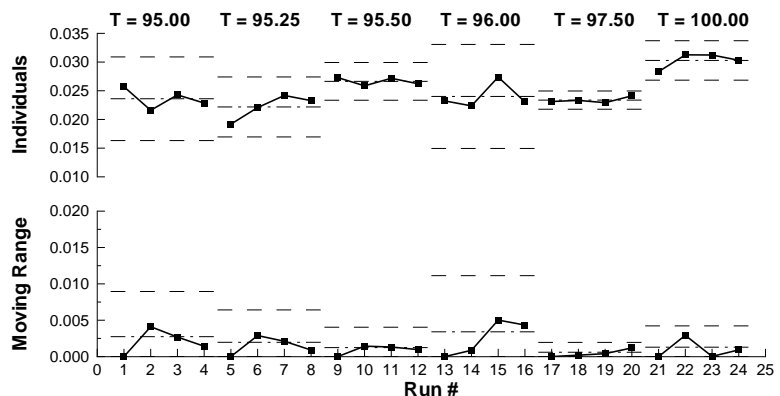

(f) $A_{4}$ Coefficient

Figure 9. Temperature Effects on Everhart Calibration Coefficients using 15-psid Transducer 
$p=A_{1} Z^{\frac{1}{3}}+A_{2} Z^{\frac{1}{2}}+A_{3} Z+A_{4} Z^{2}$

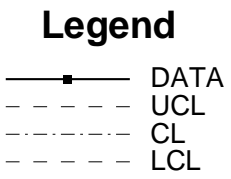

(a) Functional Form and Legend

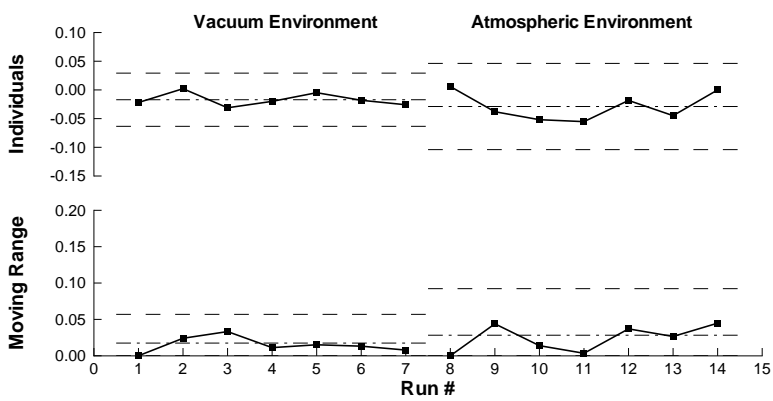

(c) $A_{1}$ Coefficient

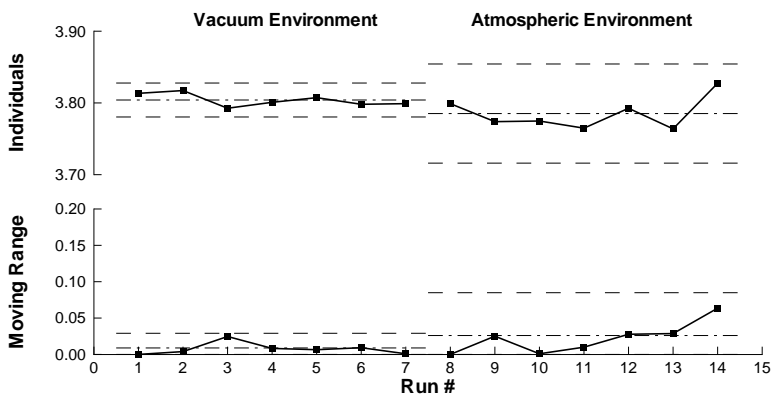

(e) $A_{3}$ Coefficient
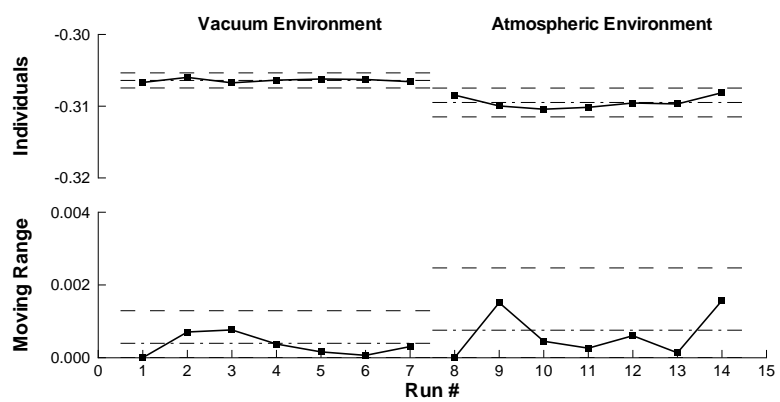

(b) $V_{0}$ Environmental Pressure Sensitivity
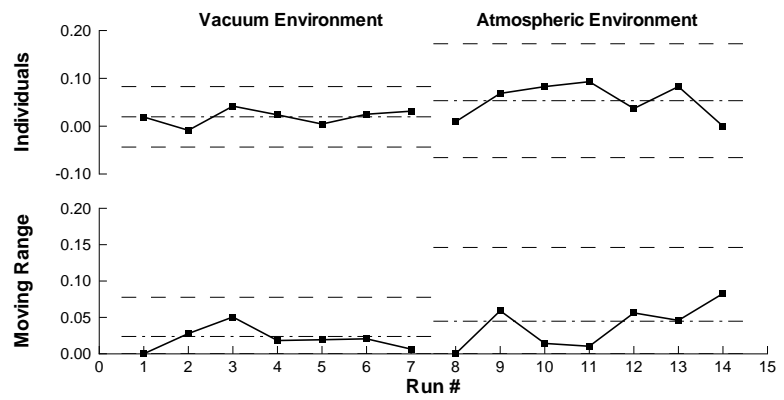

(d) $A_{2}$ Coefficient

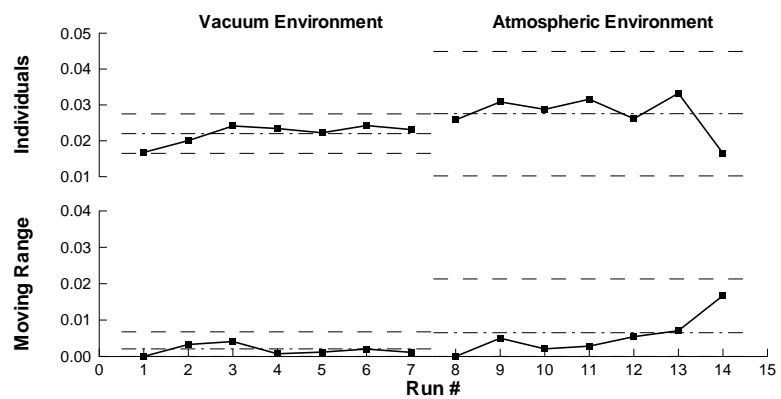

(f) $A_{4}$ Coefficient

Figure 10. Environmental Pressure Effects on Everhart Calibration Coefficients using 15 psid Transducer 
$p=C_{0}+C_{1} X^{+} C_{2} X^{2}+C_{3} X^{3}+C_{4} X^{4}$

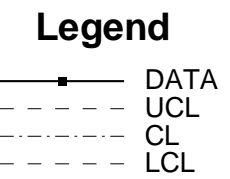

(a) Functional Form and Legend

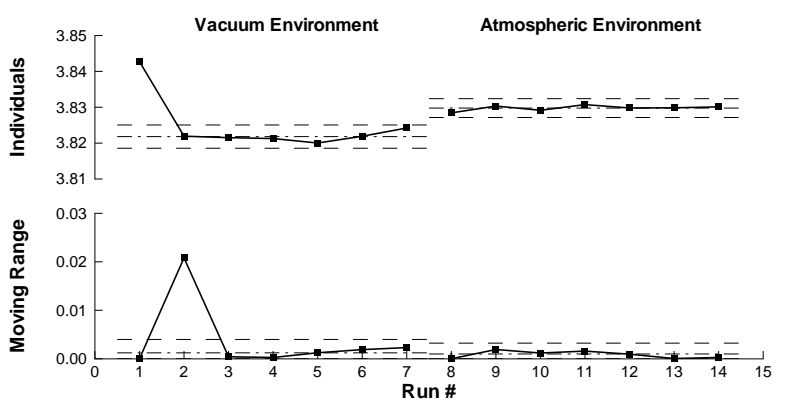

(c) $C_{1}$ Coefficient

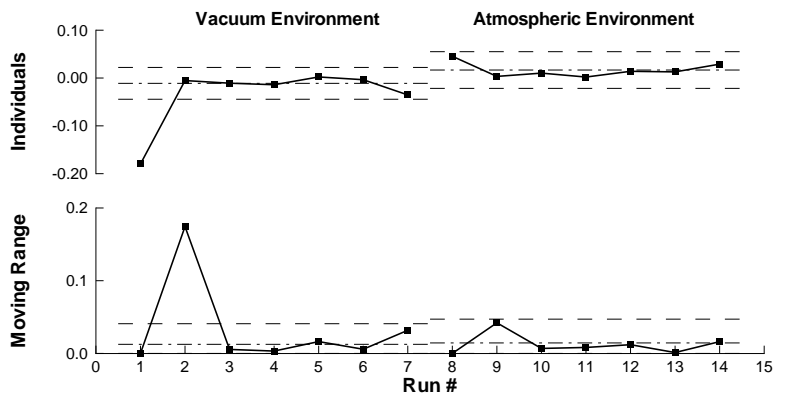

(e) $C_{3}$ Coefficient
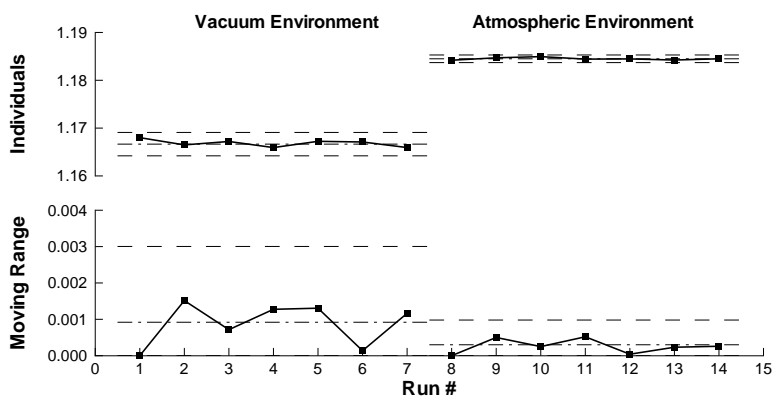

(b) $C_{0}$ Coefficient
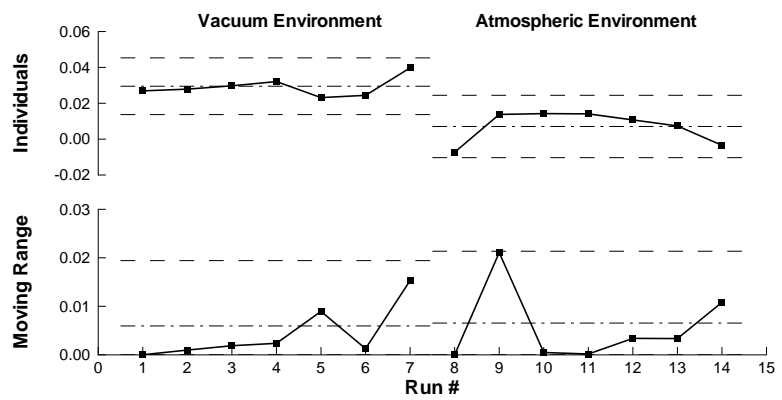

(d) $C_{2}$ Coefficient
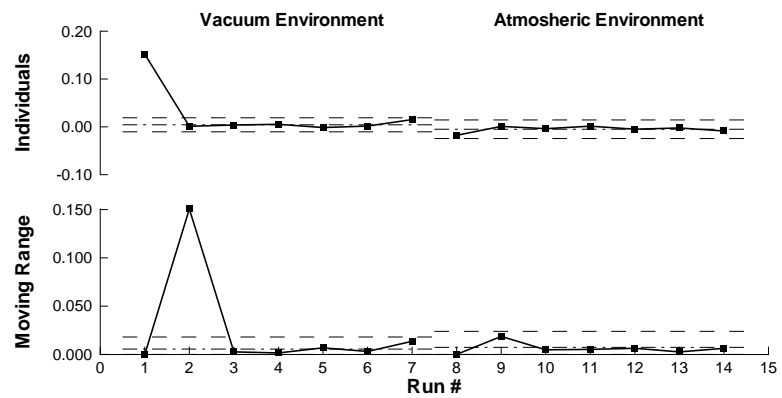

(f) $C_{4}$ Coefficient

Figure 11. Environmental Pressure Effects on PSI 4th Order Calibration Coefficients using 15 psid Transducer 
$p=A_{1} Z^{\frac{1}{3}}+A_{2} Z^{\frac{1}{2}}+A_{3} Z+A_{4} Z^{2}$

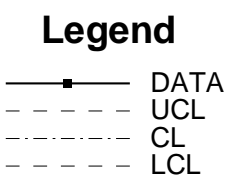

(a) Functional Form and Legend

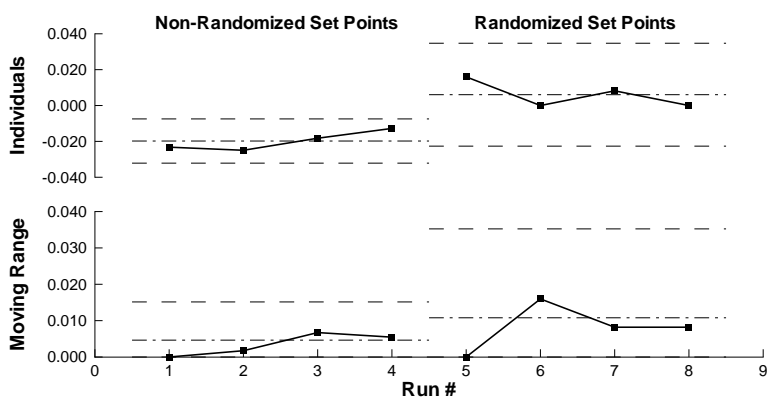

(c) $A_{1}$ Coefficient

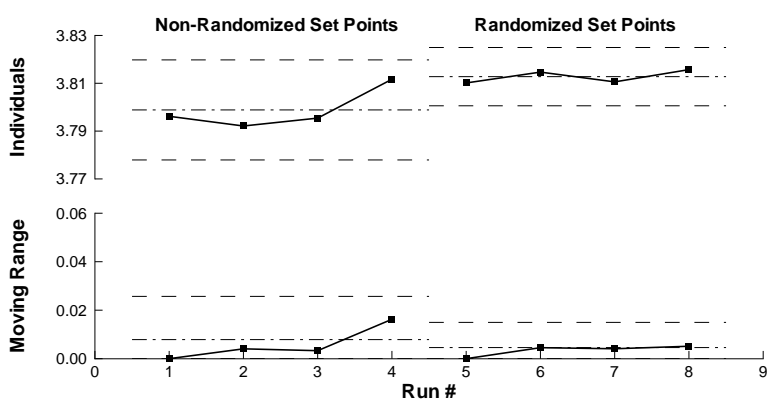

(e) $A_{3}$ Coefficient
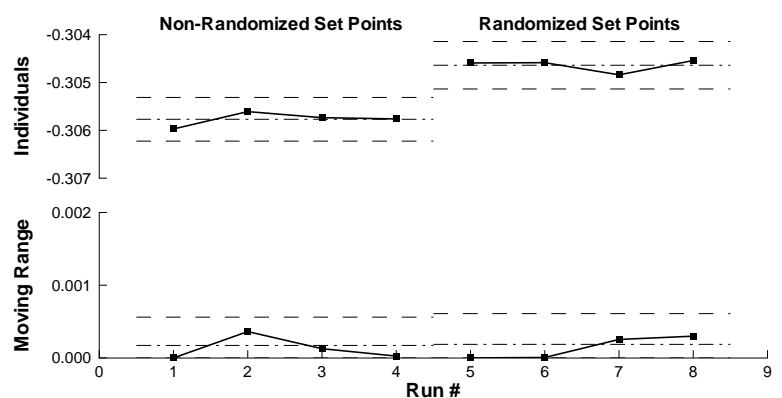

(b) $V_{0}$ Coefficient
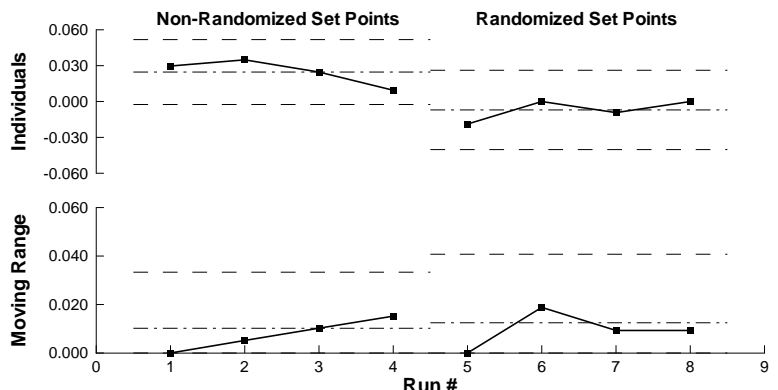

(d) $A_{2}$ Coefficient

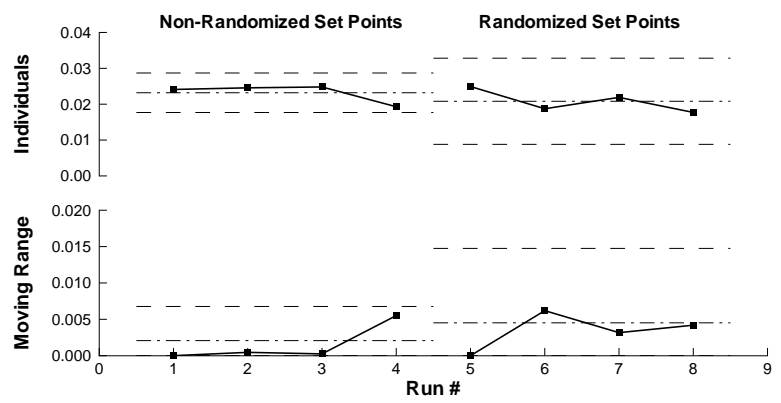

(f) $A_{4}$ Coefficient

Figure 12. Effect of Set Point Randomization on Everhart Calibration Coefficients from 15-psid Transducer Data 

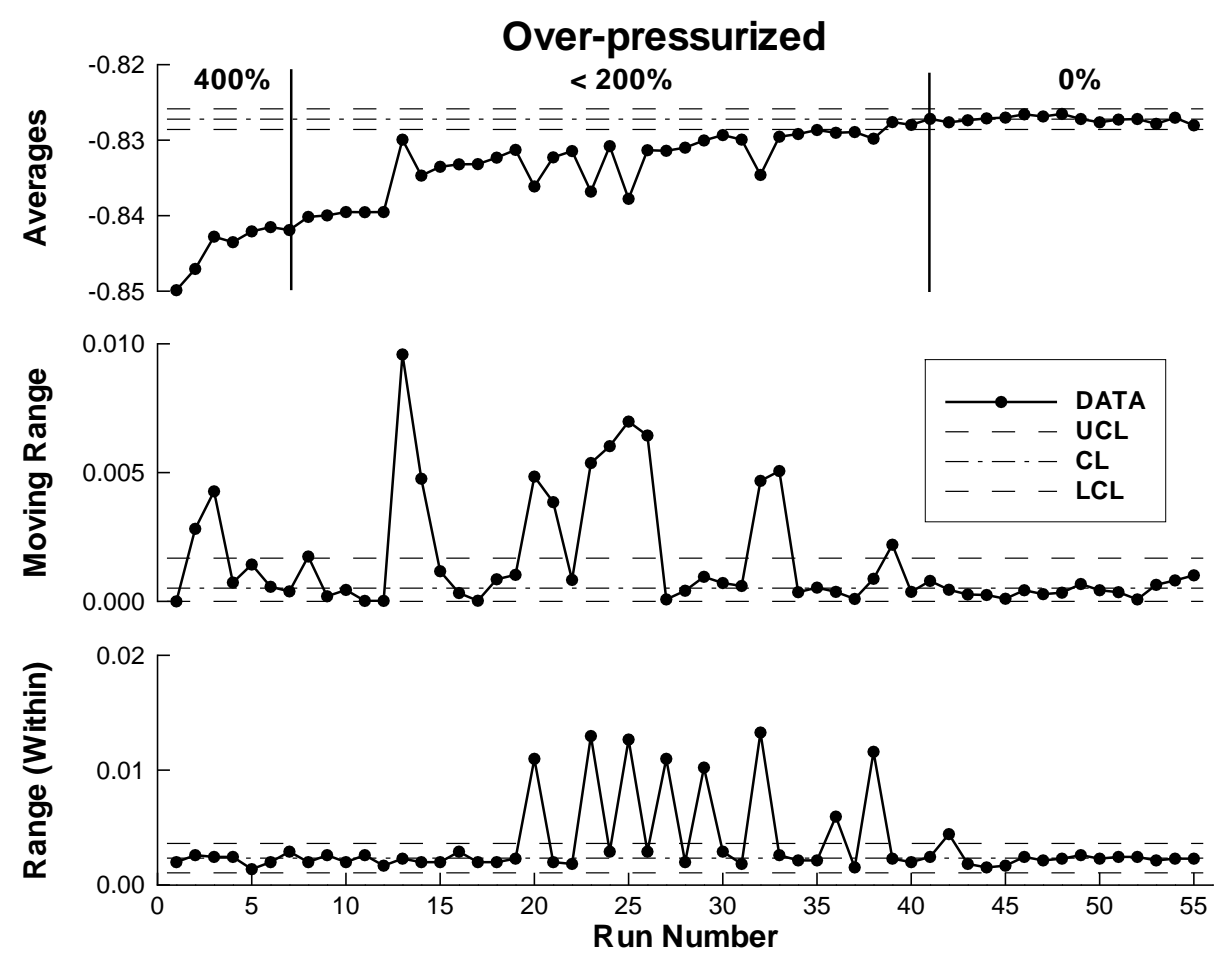

Figure 13. 0.36-psid Transducer Optimization [V]

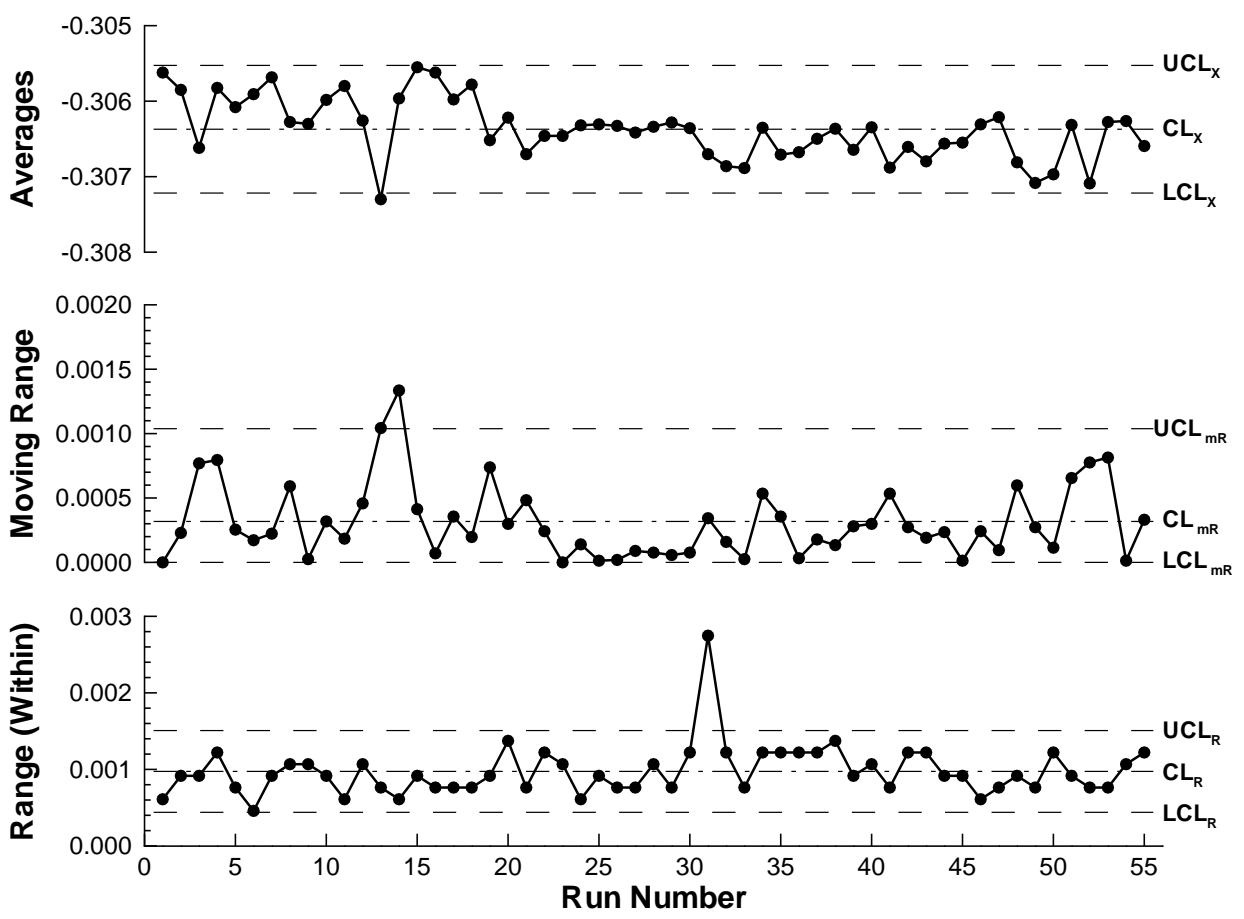

Figure 14. 15-psid Transducer Optimization [V] 
$p=A_{1} Z^{\frac{1}{3}}+A_{2} Z^{\frac{1}{2}}+A_{3} Z+A_{4} Z^{2}$

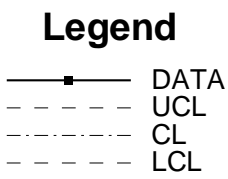

(a) Functional Form and Legend
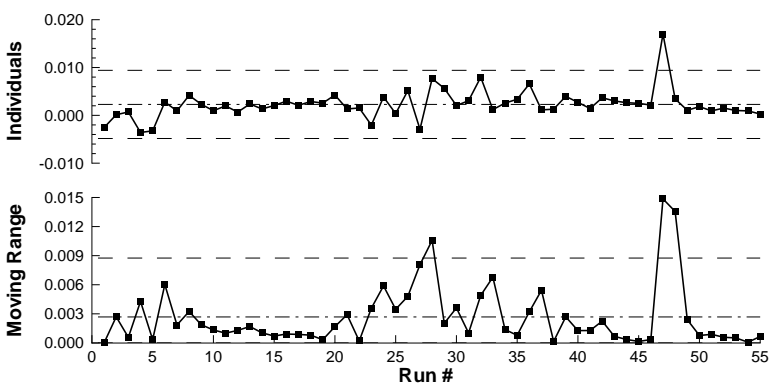

(c) $A_{1}$ Coefficients
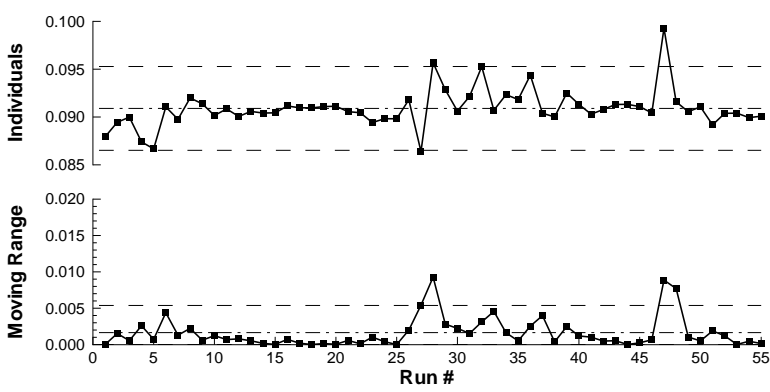

(e) $A_{3}$ Coefficients
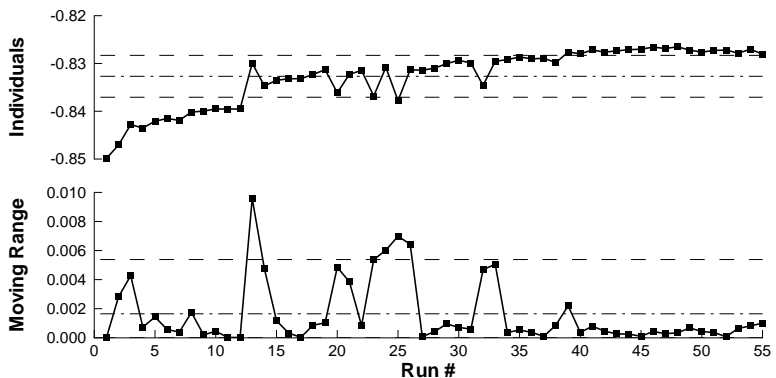

(b) $V_{0}$ Coefficients
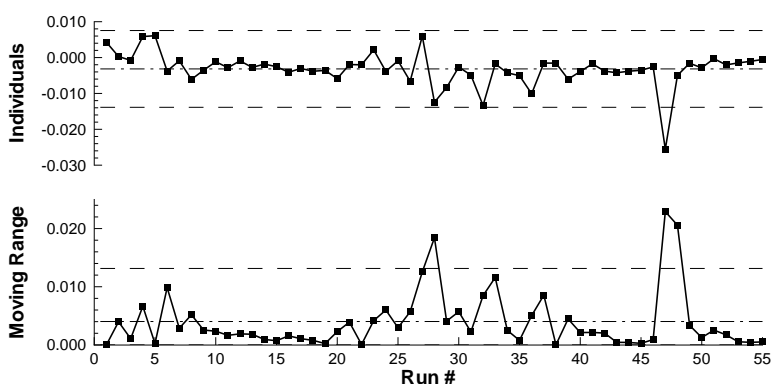

(d) $A_{2}$ Coefficients
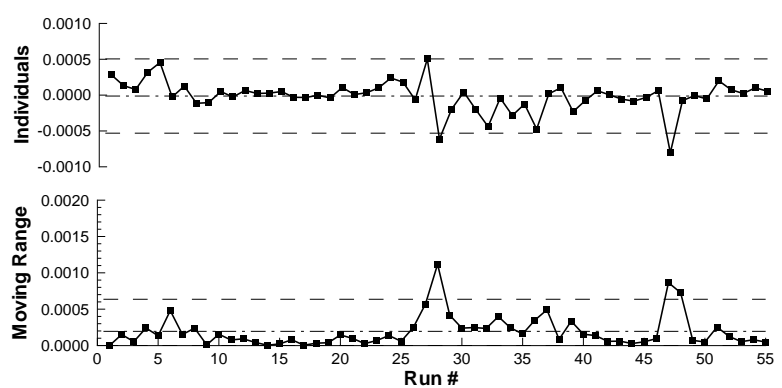

(f) $A_{4}$ Coefficients

Figure 15. Effect of Over-Pressurization on Everhart Calibration Coefficients of a 0.36-psid transducer 
$p=A_{1} Z^{\frac{1}{3}}+A_{2} Z^{\frac{1}{2}}+A_{3} Z+A_{4} Z^{2}$

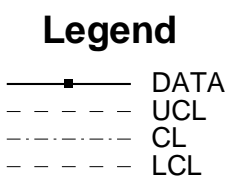

(a) Functional Form and Legend
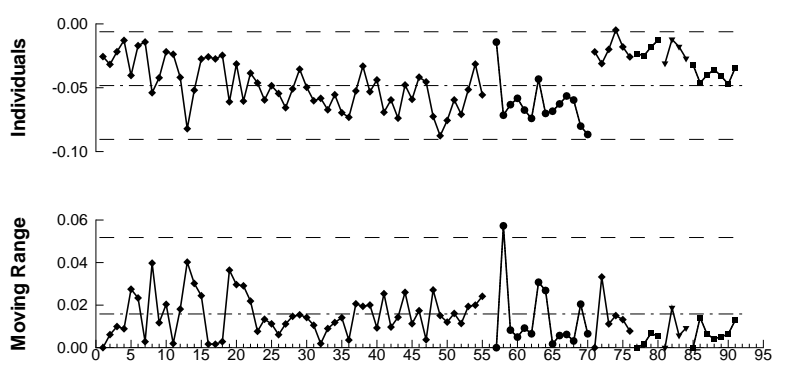

(c) $A_{1}$ Coefficients
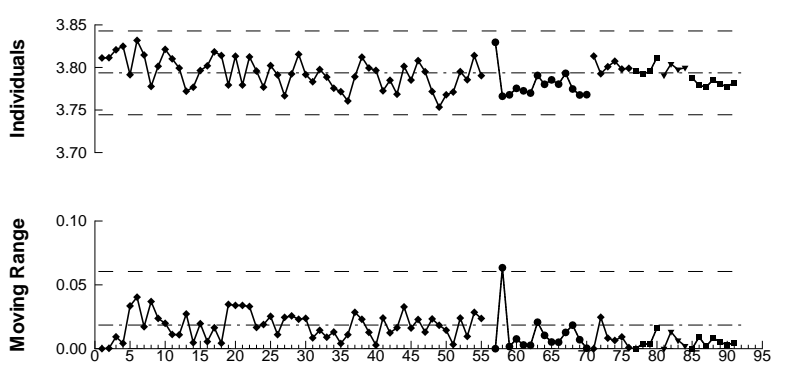

(e) $A_{3}$ Coefficients
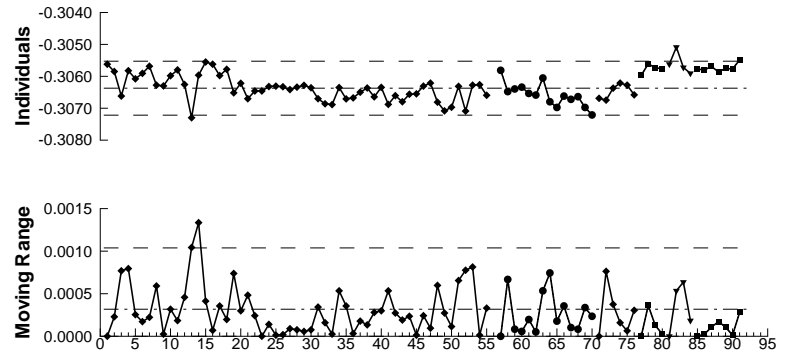

(b) $V_{0}$ Coefficients
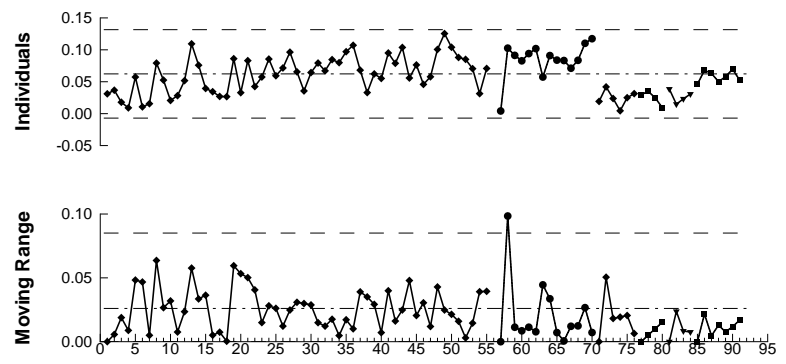

(d) $A_{2}$ Coefficients
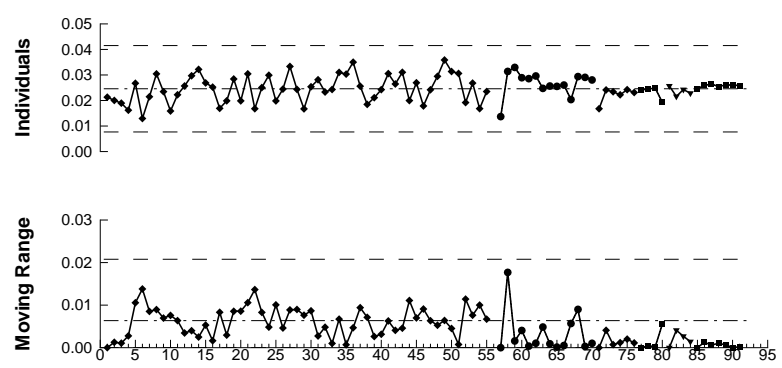

(f) $A_{4}$ Coefficients

Figure 16. Long Term Everhart Calibration Reproducibility 